ARTICLE

Received 22 Jul 2015 | Accepted 17 Jan 2016 | Published 16 Mar 2016

DOI: $10.1038 /$ ncomms10773

OPEN

\title{
Mapping the dynamics and nanoscale organization of synaptic adhesion proteins using monomeric streptavidin
}

Ingrid Chamma ${ }^{1,2}$, Mathieu Letellier ${ }^{1,2}$, Corey Butler ${ }^{1,2,5}$, Béatrice Tessier ${ }^{1,2}$, Kok-Hong Lim ${ }^{3, \dagger}$, Isabel Gauthereau ${ }^{1,2}$, Daniel Choquet ${ }^{1,2,4}$, Jean-Baptiste Sibarita ${ }^{1,2}$, Sheldon Park ${ }^{3}$, Matthieu Sainlos ${ }^{1,2, \star}$

\& Olivier Thoumine ${ }^{1,2, \star}$

The advent of super-resolution imaging (SRI) has created a need for optimized labelling strategies. We present a new method relying on fluorophore-conjugated monomeric streptavidin (mSA) to label membrane proteins carrying a short, enzymatically biotinylated tag, compatible with SRI techniques including UPAINT, STED and dSTORM. We demonstrate efficient and specific labelling of target proteins in confined intercellular and organotypic tissues, with reduced steric hindrance and no crosslinking compared with multivalent probes. We use mSA to decipher the dynamics and nanoscale organization of the synaptic adhesion molecules neurexin-1 $\beta$, neuroligin-1 (N|g1) and leucine-rich-repeat transmembrane protein 2 (LRRTM2) in a dual-colour configuration with GFP nanobody, and show that these proteins are diffusionally trapped at synapses where they form apposed trans-synaptic adhesive structures. Furthermore, Nlg1 is dynamic, disperse and sensitive to synaptic stimulation, whereas LRRTM2 is organized in compact and stable nanodomains. Thus, mSA is a versatile tool to image membrane proteins at high resolution in complex live environments, providing novel information about the nano-organization of biological structures.

\footnotetext{
${ }^{1}$ Interdisciplinary Institute for Neuroscience, UMR 5297, Centre National de la Recherche Scientifique, 33077 Bordeaux, France. ${ }^{2}$ Interdisciplinary Institute for Neuroscience, University of Bordeaux, 33077 Bordeaux, France. ${ }^{3}$ Department of Chemical and Biological Engineering, University at Buffalo, Buffalo, New York 14260, USA. ${ }^{4}$ Bordeaux Imaging Center, UMS 3420 Centre National de la Recherche Scientifique, University of Bordeaux, US 4 INSERM, 33077 Bordeaux, France. ${ }^{5}$ Imagine Optic, 18 rue Charles de Gaulle, 91400 Orsay, France. † Present address: Conagen, Bedford, Massachusetts 01730, USA. ${ }^{\star}$ These authors jointly supervised this work. Correspondence and requests for materials should be addressed to M.S. (email: sainlos@u-bordeaux.fr) or to O.T.

(email: olivier.thoumine@u-bordeaux.fr).
} 
C ritical cellular functions including adhesion and signalling are performed by dynamic macromolecular platforms at the cell membrane. Synaptic neuronal contacts are examples of such complex structures, where protein concentration is extremely high and lies in a very confined and compartmentalized space ${ }^{1}$. Recently developed fluorescencebased super-resolution imaging (SRI) techniques, including STimulated Emission Depletion (STED) $)^{2}$, PhotoActivation Localization Microscopy (PALM) $)^{3,4}$, direct STochastic Optical Reconstruction Microscopy (dSTORM) ${ }^{5,6}$ and universal Point Accumulation for Imaging in Nanoscale Topography (uPAINT) $)^{7,8}$, provide $20-50 \mathrm{~nm}$ resolution maps of single-molecule localization in biological samples, allowing a better understanding of the organization and turnover of these submicron multisubunit assemblies ${ }^{9}$. To accompany such progress in imaging power, there is a pressing need for efficient labelling strategies relying on small and penetrating probes that provide high signal-to-noise ratio and minimal linkage error with respect to target proteins ${ }^{10,11}$.

Protein labelling has relied for a long time on the use of antibodies, which have the strong advantage of targeting endogenous proteins. Yet, full-length antibodies are relatively large $(10-15 \mathrm{~nm})$ compared with nanoscale biological objects, thereby causing potential steric hindrance and localization bias ${ }^{11,12}$. Moreover, because of their divalence, antibodies may induce protein crosslinking at the cell surface. The recent expansion of antibody fragments such as $\mathrm{Fab}, \mathrm{scFv}$ or $\mathrm{VhH}$ is promising improvements ${ }^{13,14}$; however, their development including antigen preparation is time-consuming ${ }^{15}$. Alternative labelling approaches consist of the use of recombinant proteins fused to fluorescent proteins (FPs, photoactivatable or photoswitchable proteins) ${ }^{3,16}$, reactive moieties such as modified enzymes to bind small dye-labelled synthetic molecules ${ }^{17,18}$ or small peptide tags for subsequent conjugation with exogenous fluorescent ligands ${ }^{19,20}$. However, FPs usually have weaker fluorescence compared to organic dyes, and the insertion of a FP or enzyme moiety $(25-35 \mathrm{kDa})$ may affect proper protein folding and/or function ${ }^{21,22}$.

In this context, we searched for an efficient and orthogonal labelling approach combining minimal tag size on the protein of interest, probe penetrability in confined regions, absence of artefact due to multivalence and compatibility with multicolour imaging. We propose a new method based on streptavidin monomers (mSA) to deliver bright organic fluorophores to proteins that are enzymatically biotinylated on a 15-amino-acid acceptor peptide (AP) $\operatorname{tag}^{23}$. This targeted biotinylation strategy was previously developed in combination with monovalent streptavidin to track membrane molecule dynamics using quantum $\operatorname{dots}^{24}$. However, monovalent streptavidin remains a relatively large heterotetramer (molecular weight of $60 \mathrm{kDa}$ and $\sim 6 \mathrm{~nm}$ across) and is difficult to produce because of the precise 3-to-1 stoichiometry that is required among the subunits ${ }^{24}$. $\mathrm{mSA}$ is a structural monomer evolved from wild-type streptavidin tetramer with the aim of maximizing aqueous stability (denaturation temperature of $60^{\circ} \mathrm{C}$ ) while maintaining sufficient biotin affinity $\left(K_{\mathrm{d}}=2.8 \mathrm{nM}\right)^{25,26}$. The resulting $\mathrm{mSA}$ is a stable 3-nm labelling probe with a molecular weight of $12.5 \mathrm{kDa}$, which is simple to produce and to couple to various fluorophores for SRI applications.

We demonstrate that fluorescently conjugated $\mathrm{mSA}$ is compatible with a wide range of microscopy techniques, including uPAINT, Fluorescence Recovery After Photobleaching (FRAP), STED and dSTORM, in both live and fixed conditions. We further show that, unlike antibodies or streptavidin, mSA labels its targets without crosslinking and penetrates deep into live tissues. We applied mSA-based labelling to probe several synaptogenic adhesion proteins in neurons, including the presynaptic neurexin-1 $\beta(\operatorname{Nrx} 1 \beta)$, and its postsynaptic binding partners neuroligin-1 (Nlg1), and leucine-rich-repeat transmembrane protein 2 (LRRTM2), against which good antibodies compatible with live cell imaging are lacking. We demonstrate differential nanoscale organization and dynamics of Nlg1 and LRRTM2, which may underlie divergent physiological roles at the synapse.

\section{Results}

mSA labels biotinylated proteins at the cell surface. Recombinant mSA was produced in bacteria, purified by affinity chromatography via a 10 -His N-terminal tag and covalently conjugated to photostable organic dyes Atto 594 or Atto 647N for UPAINT and STED experiments, respectively, or to Alexa 647 for dSTORM imaging, using standard N-hydroxysuccinimide (NHS) ester coupling onto solvent-exposed primary amines (Supplementary Fig. 1). Fluorophore-conjugated mSA was used to label membrane proteins in neurons, COS-7 or HEK-293 cells that incorporate an extracellular AP tag. Biotinylation of the AP tag occurred during protein maturation by co-expressed Escherichia coli biotin ligase, $\operatorname{BirA}^{\mathrm{ER}}$ (ref. 17). When added to the extracellular imaging solution, the mSA-dye conjugate bound specifically to cells co-expressing AP-tagged proteins and BirA ${ }^{\mathrm{ER}}$, and not to cells expressing either haemagglutinin (HA)-tagged proteins and BirA ${ }^{\mathrm{ER}}$, or AP-tagged proteins but lacking BirA ${ }^{\mathrm{ER}}$ (Supplementary Fig. 2a-d). mSA labelling was efficient on all cell types and membrane proteins tested and produced images with high signal-to-noise ratio. The relatively fast dissociation kinetics of $\mathrm{mSA} \quad\left(k_{\mathrm{off}}=1.05 \times 10^{-3} \mathrm{~s}^{-1}\right)^{25,26}$ allow efficient release of bound mSA with excess free biotin. In this regard, addition of $200 \mu \mathrm{M}$ biotin to the labelling solution resulted in rapid loss of the mSA label (Supplementary Fig. 2e,f). Such reversible binding may be exploited to visualize internalized protein complexes using competition with modified biotin having reduced membrane permeability 27 , and synthesized for this study (Supplementary Fig. 3 and Supplementary Movie 1). Covalent labelling or labelling with high-affinity streptavidin derivatives, for example, monovalent tetramer ( $k_{\text {off }}$ $\left.\sim 4.2 \times 10^{-6} s^{-1}\right)^{17,24,28}$, would not allow such measurements. Taken together, these results demonstrate the high specificity of mSA labelling for recombinant biotinylated proteins in a cellular context.

mSA reports on protein dynamics without crosslinking. To evaluate the impact of probe valence on observed protein dynamics, we used uPAINT ${ }^{8}$ to measure the diffusion of biotinylated AP-tagged Nlg1 (AP-Nlg1) in neurons that were labelled with divalent biotin antibody, tetrameric streptavidin or mSA conjugated with Atto 594 (Fig. 1a and Supplementary Fig. 1). The three probes were added at a low concentration $(1 \mathrm{nM})$ to isolate single Nlg1 molecules diffusing on the cell surface. Neurons electroporated with AP-Nlg1 exhibited a twofold stronger surface labelling with $\operatorname{Nrx} 1 \beta-\mathrm{Fc}$ and twofold higher anti-Nlg1 signal in western blots than control neurons expressing empty vector or Homerlc-green fluorescent protein (GFP), indicating a ratio of approximately one overexpressed AP-Nlg1 molecule for one endogenous Nlg1 molecule (Supplementary Fig. 4). In immature neurons, at 7 days in vitro (7 DIV), the distribution of AP-Nlg1 diffusion coefficients after mSA labelling was centred around $0.1 \mu \mathrm{m}^{2} \mathrm{~s}^{-1}$, revealing fast Brownian motion (Fig. 1b,c). When the movement of AP-Nlg1 was tracked with Atto-conjugated biotin antibody or tetrameric streptavidin, the measured diffusion coefficients were shifted towards lower values (Fig. 1b,c) and there was a concomitant 
a

Monomeric streptavidin $(\mathrm{mSA})$

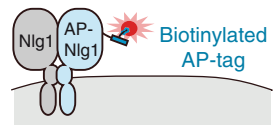

b

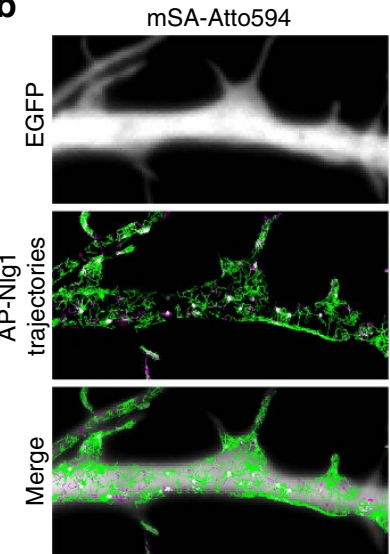

e
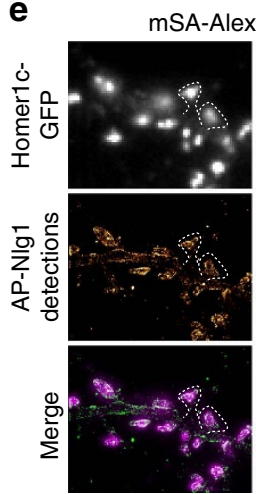
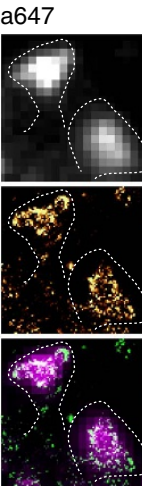

Anti-biotin antibody

(Ab)

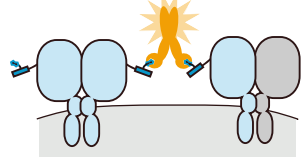

Ab-Atto594
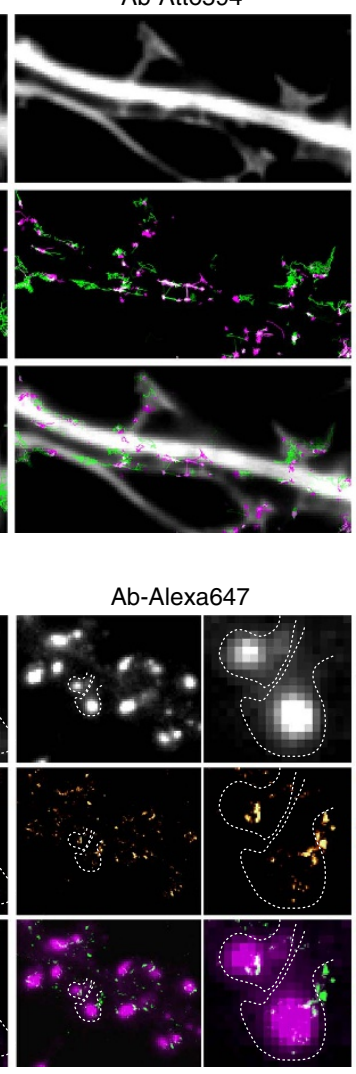

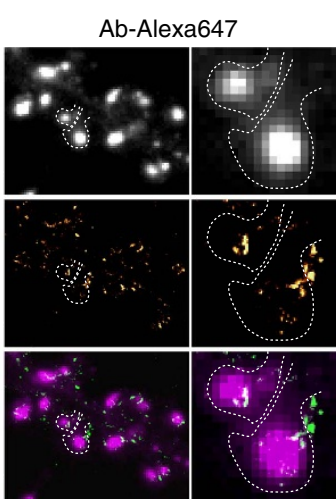

Tetrameric streptavidin (Strept)

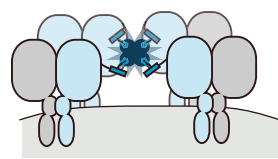

Strept-Atto594

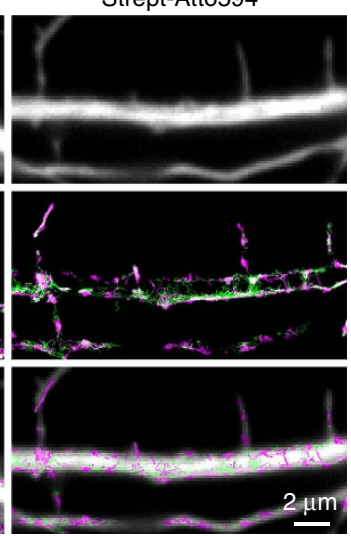

Strept-Alexa647

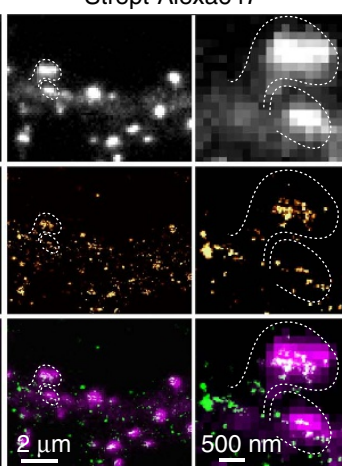

C

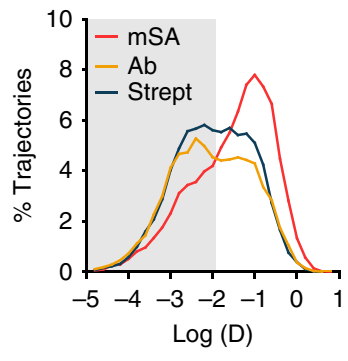

d
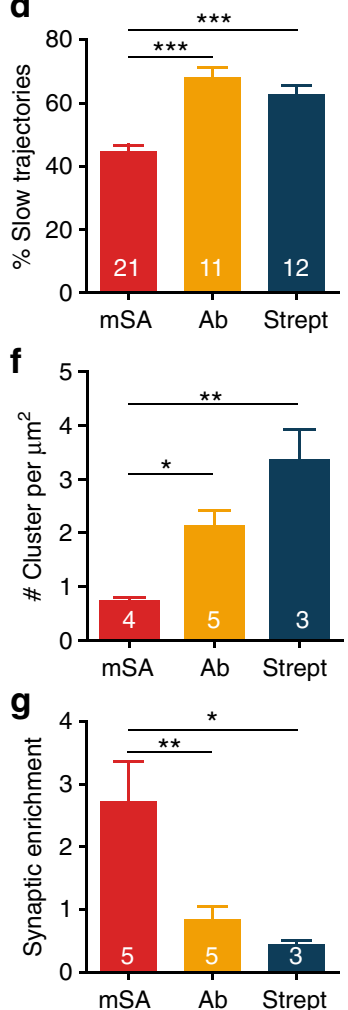

Figure 1 | Super-resolution imaging of AP-Nlg1 with mSA or biotin antibody or tetrameric streptavidin. (a) Schematic diagram of AP-Nlg1 labelled with three different probes (mSA, monoclonal biotin antibody or streptavidin), conjugated to Atto 594 for uPAINT or Alexa 647 for dSTORM. (b) Examples of DIV 7 neurons co-expressing EGFP as a volume marker, AP-NIg1 and BirA ${ }^{E R}$, and labelled as described above. Middle panels display AP-NIg1 trajectories

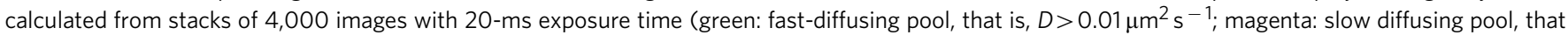
is, $D<0.01 \mu \mathrm{m}^{2} \mathrm{~s}^{-1}$ ). Merged images show EGFP (grey) overlaid with AP-Nlg1 trajectories. Note that mSA explores larger surface areas with faster diffusion than multivalent ligands. (c) Distribution of AP-Nlg1 diffusion coefficients in a semi-log plot, where the grey-shaded area represents slow

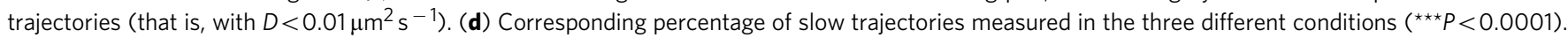
Data are from three different experiments. (e) Examples of DIV 15 neurons co-expressing Homer1c-GFP, AP-NIg1 and BirA ER, and labelled with the three Alexa647-conjugated probes shown in a. Top panels: Homer1c-GFP signal showing mature synapses (white). Middle panels: super-resolved AP-Nlg1 detection maps generated from 40,000 frames with 20-ms integration time. Bottom panels: merged images showing AP-Nlg1 detections (green) overlaid with Homer1c-GFP (magenta). Note the presence of large AP-NIg1 aggregates in anti-biotin and streptavidin-labelled neurons. (f) Histogram showing the number of $\mathrm{Nlg} 1$ clusters per $\mu \mathrm{m}^{2}$ in the three conditions ( $\left.{ }^{\star \star} \mathrm{P}<0.01\right)$. (g) Histogram showing the synaptic enrichment of AP-Nlg1 compared with the shaft $\left({ }^{\star \star} P<0.01,{ }^{\star} P<0.05\right)$. Data are from two different experiments. Numbers in the bar charts represent the number of cells examined.

increase in the fraction of slowly diffusing molecules (Fig. 1d), defined as $D<0.01 \mu \mathrm{m}^{2} \mathrm{~s}^{-1}$ (Supplementary Fig. 5). The surface area covered by AP-Nlg1 trajectories was also higher with mSA than with biotin antibody or streptavidin (Fig. 1b). Together, these results suggest that divalent and tetravalent probes alter AP-Nlg1 distribution and dynamics through a combination of protein crosslinking and steric hindrance.

mSA prevents aggregation compared with multivalent probes. To examine the effects of probe size and valence on Nlg1 distribution at the nanoscale, we used ASTORM to image Nlg1 in mature neurons (DIV 15) 5,29,30. Membrane-bound AP-Nlg1 was labelled with $100 \mathrm{nM} \mathrm{mSA}$, biotin antibody or streptavidin conjugated with Alexa 647 for $10 \mathrm{~min}$ before fixation and observation. In contrast to our UPAINT experiments, which rely on sparse labelling and short acquisition sequences to focus on protein dynamics, dSTORM uses high-density labelling combined with long acquisition times $(800 \mathrm{~s})$ to produce a static representation of the overall protein organization. In parallel, postsynaptic densities were identified using co-expressed Homer1c-GFP ${ }^{31,32}$. mSA labelling was fairly diffuse in the shaft and highly concentrated at synapses (Fig. 1e). In contrast, both biotin antibody and streptavidin induced the aggregation of 
AP-Nlg1 into numerous small clusters on dendrites (Fig. 1e,f), which are most likely caused by ligand-mediated receptor crosslinking. The anti-biotin-labelled AP-Nlg1 was found mostly on the edge of the synapses around rather than inside, presumably because the antibody has limited accessibility to the synaptic cleft because of its large size. With its intermediate size, tetrameric streptavidin penetrated well inside synapses, but because of massive extrasynaptic clustering was less enriched than anti-biotin at synapses compared with shaft levels. Overall, the enrichment of AP-Nlg1 at Homer1c-GFP-positive puncta was significantly higher for mSA compared with streptavidin and antibody (Fig. 1g), indicating that mSA enables a more accurate visualization of receptor localization and organization at the nanometre scale.

mSA efficiently labels Nlg1 in the confined synaptic cleft. To assess the ability of mSA-labelled Nlg1 to dynamically access the synaptic cleft ( $20 \mathrm{~nm}$ across), we performed uPAINT experiments on live DIV 10-15 neurons expressing AP-Nlg1, and labelled using either mSA or biotin antibody conjugated to Atto594. Using mSA, we observed a gradual decrease in AP-Nlg1 diffusion across neuronal development (Supplementary Fig. 6a-c), revealing the stabilization of Nlg1 associated with an increase in synapse density and maturation ${ }^{33}$. On DIV 15, AP-Nlg1 trajectories recorded with mSA populated both the dendritic shaft and synapses and substantially overlapped with the synaptic Homerlc-GFP staining (Fig. 2a and Supplementary Movie 2), whereas AP-Nlg1 trajectories obtained with biotin antibody were mostly localized on the dendritic shaft and confined to areas around the postsynaptic density. Quantitatively, 85\% of synapses contained mSA-labelled Nlg1, while only $40 \%$ contained antibody-labelled Nlg1 (Fig. 2b), and the synaptic area covered by mSA-labelled Nlg1 was fourfold higher than antibody-labelled Nlg1 (30\% versus $8 \%$, respectively; Fig. 2c). mSA-labelled Nlg1 exhibited fast diffusion in extrasynaptic compartments and slower diffusion at synapses (Fig. 2d), reflecting interactions with presynaptic axonal adhesion protein, Nrx and/or postsynaptic scaffolding proteins ${ }^{32}$. In contrast, anti-biotin labelling showed consistently slower diffusion across the entire neuronal surface, similar to the observations on DIV 7 (Fig. 1c). An additional level of immobilization of mSA-labelled AP-Nlg1 on DIV 15 was observed on incubation with soluble dimeric Nrx1 $\beta$-Fc (Supplementary Fig. 6d,e), which is consistent with the preferential anchorage of Nlg1 to synaptic PSD-95 triggered by Nrx1 $\beta$ binding $^{32}$. These observations show that $\mathrm{mSA}$ can be used to label both fast- and slow-moving AP-Nlg1 in developing neurons, and to visualize trapping events in mature synapses without introducing steric and valence bias compared with divalent antibodies.

mSA labels target proteins in organotypic brain tissue. Given the small size and high labelling efficiency of mSA, we tested its capability to label neurons in dense organotypic tissue. We co-expressed GFP, AP-Nlg1 and BirA ${ }^{E R}$ in individual CA1 pyramidal neurons from $150-\mu \mathrm{m}$-thick mouse organotypic a
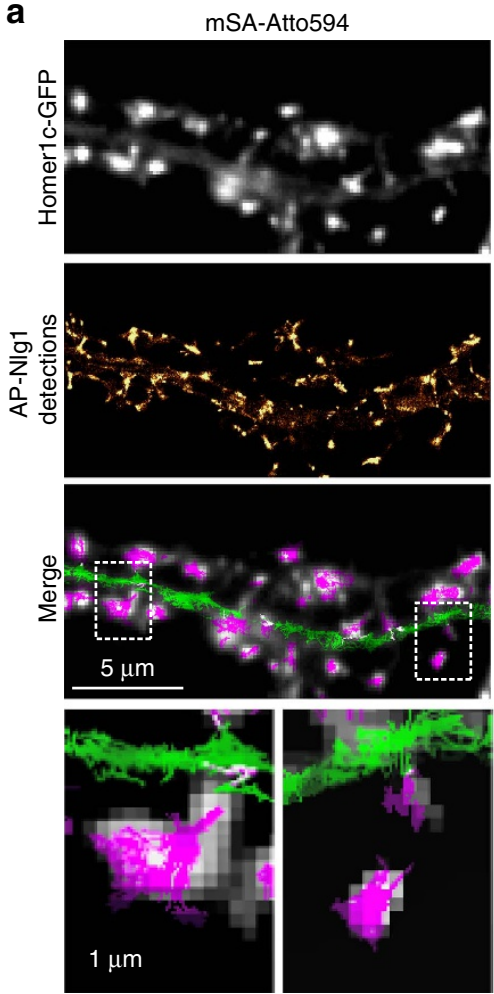
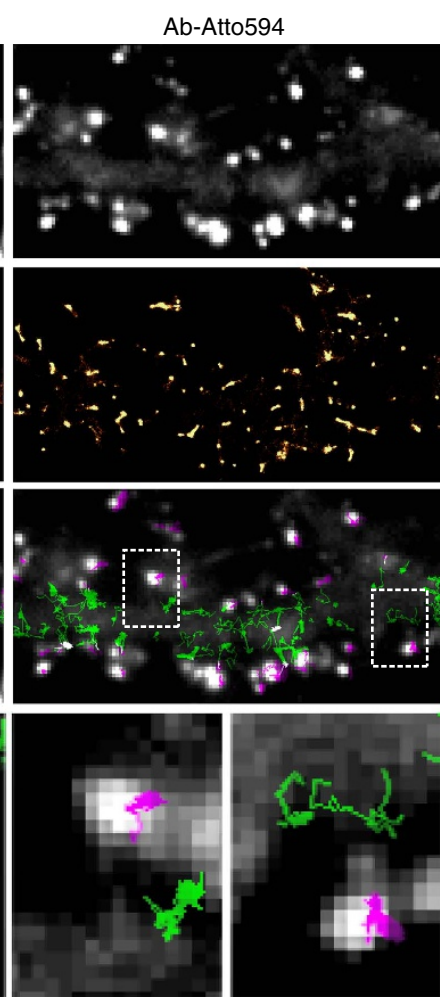

b

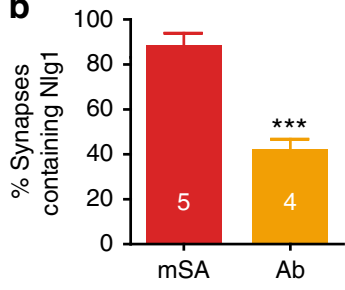

C

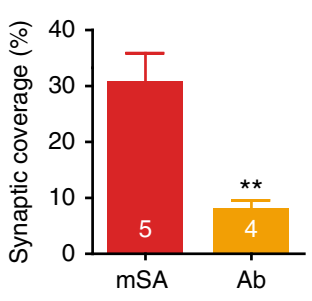

d

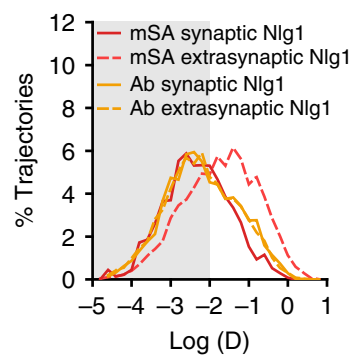

Figure 2 | Different ability of mSA and biotin antibody to label AP-Nlg1 within the synaptic cleft in live conditions. (a) DIV 15 neurons expressing Homer1c-GFP, AP-NIg1 and BirA ${ }^{\text {ER }}$ were labelled with mSA or anti-biotin conjugated to Atto594 to track individual AP-NIg1 molecules by uPAINT. From top to bottom: Homer1c-GFP signal staining mature synapses; super-resolved AP-Nlg1 detection maps; merged images showing extrasynaptic AP-Nlg1 trajectories (green) and synaptic trajectories (magenta) overlaid with Homer1c-GFP signals (grey); insets show that mSA-stained Nlg1 fills the entire synaptic area, whereas antibiotin remains on the edge of the postsynaptic density. (b) Percentage of synapses containing AP-Nlg1 labelled with mSA or biotin antibody ( $\left.{ }^{\star \star \star} P<0.0001\right)$. (c) Percentage of the synaptic area occupied by AP-Nlg1 when labelled with mSA or anti-biotin ( $\left.{ }^{\star \star P}<0.01\right)$. (d) Semi-log distribution of synaptic (solid lines) and extrasynaptic (dashed lines) diffusion coefficients for AP-Nlg1 measured with the two different probes. Data are from two different experiments. Numbers in the bar charts represent the number of cells analysed. 
hippocampal slices using single-cell electroporation. Slices were labelled by one-step incubation with $100 \mathrm{nM} \mathrm{mSA-Atto} 647 \mathrm{~N}$ (for STED) or mSA-Alexa647 (for dSTORM), then rinsed and observed live (STED) or after fixation (dSTORM). The mSAlabelling of AP-Nlg1 was highly specific since it stained only GFPco-expressing cells throughout the slice with extremely low background, and localized exclusively to somatodendritic regions (Fig. 3a,b and Supplementary Movie 3). The photostability of Atto dyes combined with the thermal stability of $\mathrm{mSA}$ allowed an extensive live STED imaging of optical sections at $37^{\circ} \mathrm{C}$ down to $40 \mu \mathrm{m}$ deep. Zoom on dendritic portions showed specific enrichment of Nlg1 in spines compared with GFP (Fig. 3c,d and Supplementary Movie 4), which is also observed in dissociated hippocampal cultures (Fig. 1e-g).

Using conventional confocal microscopy, we evaluated how well mSA penetrates into brain slices compared with streptavidin and biotin antibodies. $\mathrm{mSA}$ and tetrameric streptavidin were able to label AP-Nlg1 expressed in live electroporated neurons within a few minutes, although the labelling efficiency of streptavidin decreased rapidly with depth, suggesting an impact of the probe size and/or valence in reaching deep tissues (Supplementary Fig. 7a,b). At the same sample depth $(\sim 15 \mu \mathrm{m}$ below the surface of the slice), the anti-biotin signal was not detectable after a 10-min live labelling, but became apparent after $1 \mathrm{~h}$ (Supplementary Fig. 7c). Finally, we used astigmatism-based three-dimensional (3D) dSTORM to image the distribution of mSA-labelled AP-Nlg1 in small dendritic regions on the surface of a slice from Nlg1 knockout (KO) mice, revealing high enrichment of Nlg1 in spines (Fig. 3e,f and Supplementary Movie 5). These results show that $\mathrm{mSA}$ penetrates deep into thick tissues, where it can be used to label target proteins at high density and with specificity for immediate application in SRI. Interestingly, a similar AP-Nlg1 localization was observed in STED images made on organotypic slices from wild-type mice, and in STORM images made on organotypic slices from Nlg1 KO mice, where AP-Nlg1 expressed in CA1 cells replaces endogenous Nlg1. a
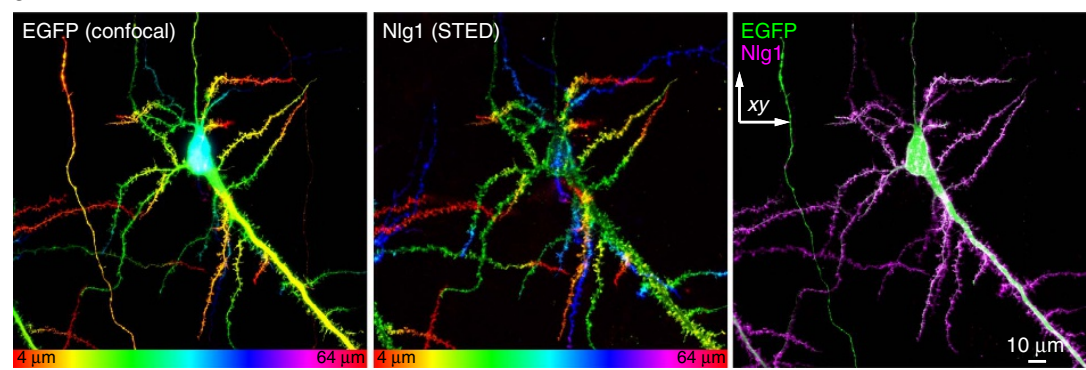

C
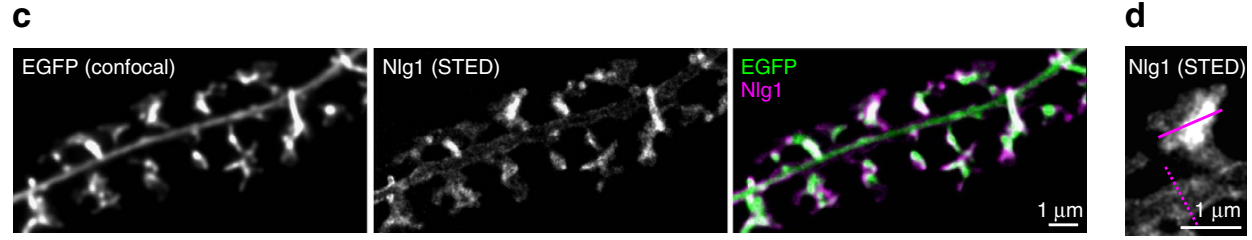

b

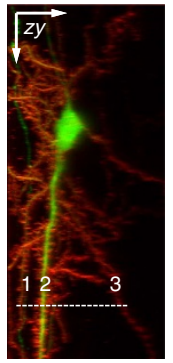

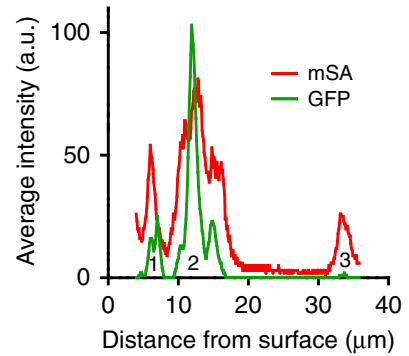

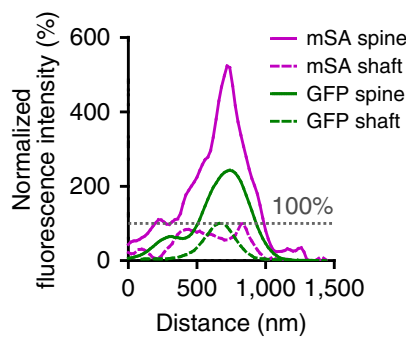

$\mathbf{f}$
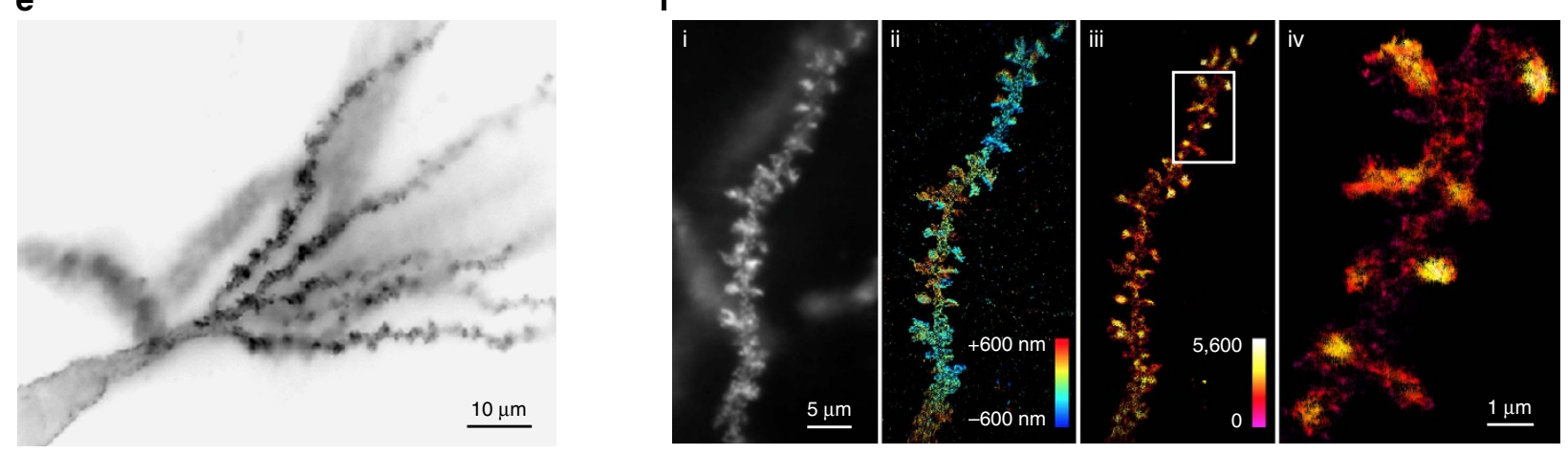

Figure 3 | Super-resolution imaging of AP-Nlg1 in organotypic hippocampal slices. (a) Confocal (EGFP, left), STED (mSA-Atto 647N, middle) and merged (right) images acquired from live neurons expressing GFP, AP-Nlg1 and BirA ${ }^{E R}$ in organotypic hippocampal slices. Images are projections of a $z$-stack of 60 planes taken by $1-\mu \mathrm{m}$ increments and colour-coded with respect to sample depth. (b) Linescan measurements of mSA-Atto 647N and GFP staining along the $z$ axis. The reduction in $\mathrm{mSA}$-Atto $647 \mathrm{~N}$ intensity with the sample depth likely reflects reduced laser penetration rather than weaker staining, since the GFP signal also decreases with depth. (c) High-magnification deconvoluted STED projection of mSA-labelled Nlg1 in a hippocampal slice shows the accumulation of Nlg1 at dendritic spines. (d) Linescans of GFP and mSA-Atto 647N fluorescence intensity in the shaft membrane and in a dendritic spine normalized to the respective fluorescence of GFP and Atto 647N in the shaft. (e) Wide field image of mSA-Alexa647 selectively labelling one neuron expressing GFP, AP-Nlg1 and BirA ${ }^{E R}$, a few microns deep from the surface of an organotypic brain slice. (f) Astigmatic-based 3D dSTORM imaging in an organotypic brain slice from Nlg1 KO mice: (i) wide field image of mSA-Alexa647-labelled AP-Nlg1. (ii) 3D dSTORM-reconstructed image of a dendritic segment based on 1,038,506 single-molecule localizations from 64,000 images. The image is colour-coded with respect to the $z$ distance $(-600$ to $+600 \mathrm{~nm}$ ). (iii) Normalized localization detection maps integrated within $z= \pm 400 \mathrm{~nm}$. (iv) Magnified view of iii showing the enrichment of Nlg1 in dendritic spines. 
mSA and GFP nanobody similarly probe protein diffusion. Monomeric VhH antibodies (nanobodies) against GFP and red fluorescent protein have been previously used for SRI, where their small size provides smaller linkage error relatively to the target structure $^{13,14}$. We thus sought to compare mSA to these well-characterized tools. To this end, we labelled the same axonal molecular target, Nrx1 $\beta$, using both approaches and compared the measured diffusion dynamics and membrane organization. On the one hand, we labelled Nrx1 $\beta$ carrying a biotinylated N-terminal AP tag with mSA-Atto594. On the other hand, Nrx1 $\beta$ fused with an N-terminal GFP tag was labelled with Atto594-conjugated GFP nanobody, resulting in a specific enrichment at excitatory pre-synapses immunostained for the vesicular transporter VGlut1 (Fig. 4a). Single-molecule trajectories were then recorded for each labelling scheme using uPAINT in DIV 15 neurons (Fig. 4b,c). The distributions of diffusion coefficients were very similar for the two probes (Fig. 4d), with a major fast diffusion peak centred around $\sim 0.5 \mu \mathrm{m}^{2} \mathrm{~s}^{-1}$, representing free diffusion in extrasynaptic compartments, and a minor slower diffusion peak around $0.005 \mu \mathrm{m}^{2} \mathrm{~s}^{-1}$, representing $\operatorname{Nrx1} \beta$ confined at presynaptic terminals ${ }^{34,35}$. These results show that, in agreement with their small size and monomeric nature, mSA and nanobody labelling lead to similar diffusion measurements. Given their orthogonality, the two approaches may thus be combined for dual-colour SRI.

The AP tag can be inserted into small extracellular loops. An advantage of using $\mathrm{mSA}$ is that the short 15-amino-acid AP tag can be readily incorporated into extracellular protein domains, such as protein loops, without perturbing the native function. To demonstrate the use of the AP tag in a system that may not be amenable for GFP fusion, we inserted the AP sequence in the first extracellular loop of the alpha-amino-3-hydroxy-5methyl-4-isoxazole-propionic acid (AMPA) receptor auxiliary protein stargazin (AP-Stg) ${ }^{36}$ (Fig. 4e). Using mSA-Atto594, we observed the localization and dynamics of AP-Stg in DIV 15 neurons by uPAINT (Fig. 4f). AP-stargazin localized at synapses where its diffusion was reduced, consistent with previously published work $^{37}$ (Fig. 4f,g). Thus, the mSA-labelling strategy can be successfully applied to situations where the use of a GFP tag may not be allowed for structural or functional reasons. To further illustrate this point, we inserted the mCherry
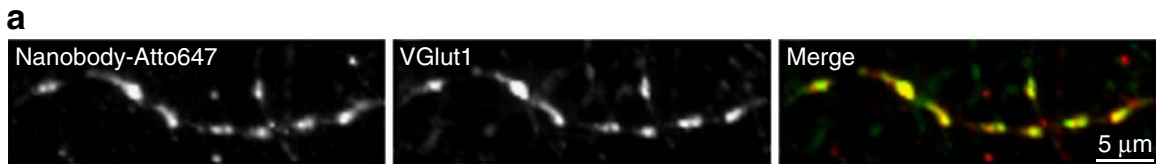

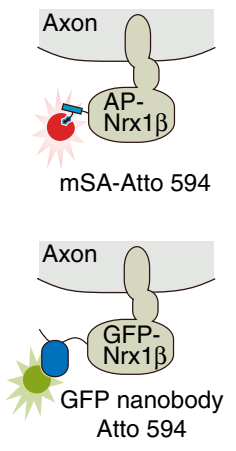

e
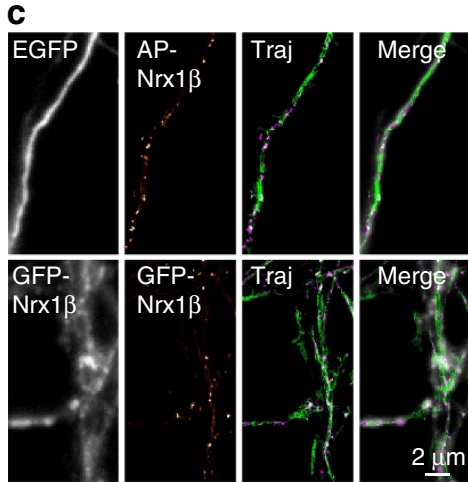

$f$

mSA-Atto 594

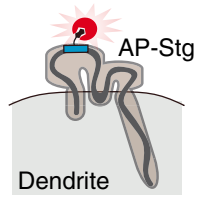

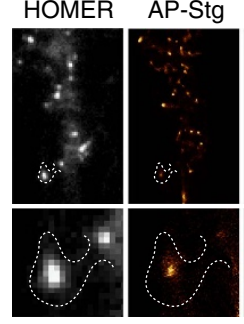

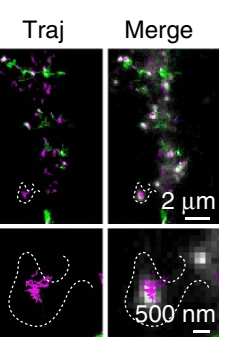

d

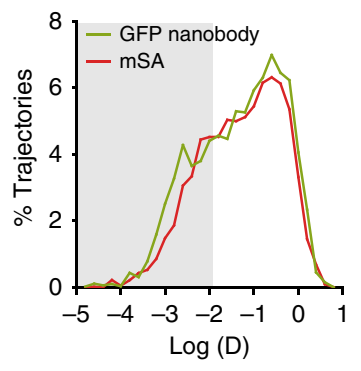

g

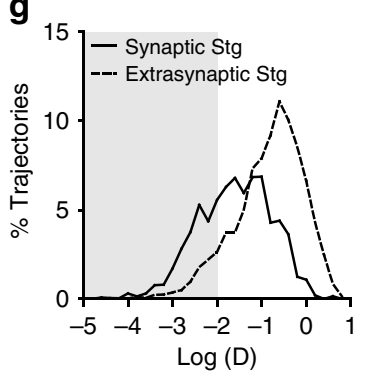

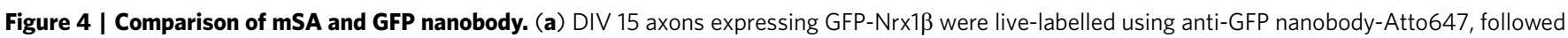

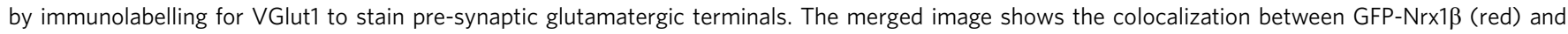
VGlut1 (green). (b) Schematics showing the labelling of AP-Nrx1 $\beta$ with mSA-Atto594 (top) and GFP-Nrx1 $\beta$ with GFP nanobody-Atto594 (bottom). (c) Examples of axonal regions from DIV 15 neurons expressing EGFP, AP-Nrx1 $\beta$ and BirA ER (top); or GFP-Nrx1 $\beta$ (bottom). From left to right: GFP signal, Nrx1 $\beta$ detection maps, colour-coded Nrx1 $\beta$ trajectory maps (green: fast-diffusing pool, that is, $D>0.01 \mu m^{2} s^{-1}$; magenta: slow diffusing pool, that is, $D<0.01 \mu \mathrm{m}^{2} \mathrm{~s}^{-1}$ ), merged image showing Nrx1 $\beta$ trajectories overlaid with GFP (grey). (d) Distributions of the diffusion coefficients obtained for AP-Nrx1 $\beta$ or GFP-Nrx1 $\beta$ ( $m S A, n=6$; GFP nanobody, $n=5$ cells from two different experiments). (e) Schematics showing the structure of the AMPA receptor auxiliary protein stargazin (Stg) with the insertion of an AP tag in the first extracellular protein loop. (f) Example of DIV 15 neurons co-expressing the synaptic marker Homer1c-GFP, AP-Stg and BirA ER. AP-Stg individual molecules were tracked using mSA-Atto594 by uPAINT. Super-resolved AP-Stg localization and trajectory maps were reconstructed from 4,000 images of 20-ms exposure time, with the same colour code as in $\mathbf{b}$. (g) Diffusion coefficient distribution of AP-Stg inside and outside synapses ( $n=6$ cells from two different experiments). 
FP (256 amino acids) in the extracellular loop of stargazin in place of the AP tag (15 amino acids). When expressed in heterologous COS-7 cells, this fusion protein formed numerous vesicular aggregates enriched in a perinuclear area, suggesting that mCherry-stg is retained in intracellular transport compartments, and not properly expressed at the cell membrane, possibly because of protein misfolding (Supplementary Fig. 8). In contrast, fusing mCherry to the $\mathrm{C}$ terminus of stargazin resulted in very clear membrane localization. This experiment clearly shows that AP tags can be advantageous compared with large domains such as FPs for the tagging of complex membrane proteins.

Dual-colour imaging of trans-synaptic adhesive contacts. Postsynaptic Nlg1 interacts with presynaptic Nrx1 $\beta$ to form trans-synaptic contacts ${ }^{38}$. To visualize the dynamic organization of mature $\mathrm{Nrx} 1 \beta / \mathrm{Nlg} 1$ complexes, we used $\mathrm{mSA}$ in combination with GFP nanobody ${ }^{13,14}$ for dual-colour uPAINT analysis. Neurons expressing either AP-Nlg1 or a blue fluorescent protein Nrx1b fusion (BFP-Nrx1 $\beta$ ) were co-cultured for 2 weeks, and the individual AP-Nlg1 and BFP-Nrx1 $\beta$ molecules on the surface of dendrites and axons, respectively, were labelled with mSA-Atto 594 and GFP nanobody-Atto $647 \mathrm{~N}$ for tracking (Fig. 5a,b and Supplementary Movie 6). Both BFP-Nrx1 $\beta$ and AP-Nlg1 showed decreased diffusion at Homer1c-GFP-positive areas, resulting in an increase in the slowly moving fraction (Fig. 5b,c), which is consistent with the formation of functional trans-synaptic complexes. The images reconstructed from all single-molecule detections indicate that every synapse contains one to two domains of Nrx1 $\beta$ and Nlg1 hot spots facing each other (Fig. 5d), with an average diameter of $85 \pm 3 \mathrm{~nm}$ for Nrx1 $\beta$ and $91 \pm 2 \mathrm{~nm}$ for $\mathrm{Nlg} 1$ (Fig. 5e,f). Given the dilute labelling conditions, these domains reflect the trapping of a small number of molecules at the synapse, whose intensity compared with diffusive molecules is amplified by the temporal integration of long-emitting fluorescent signals coming from the same location. Indeed, the domain size is in agreement with the confinement diameter measured from the mean squared displacement of synaptic Nlg1 molecules (Supplementary Fig. 5).

Addition of ethylene glycol tetra-acetic acid (EGTA) as a calcium chelator decreased the number of synaptic Nrx1 $\beta$ and Nlg1 confinement domains (Fig. 5d) and increased the diffusion of both proteins (Supplementary Fig. 9). Both changes are likely caused by a dissociation of calcium-dependent Nrx1 $\beta / \mathrm{Nlg} 1$ bonds $s^{39}$, followed by lateral motion of the two proteins. We also used dual-colour uPAINT to examine the dynamics of Nrx $1 \beta / \mathrm{Nlg} 1$ adhesion in response to synaptic stimulation in live conditions. We treated neurons with $20 \mu \mathrm{M} \mathrm{N}$-methyl-D-aspartate (NMDA) for $10 \mathrm{~min}$ to chemically induce synaptic depression ${ }^{40}$ and monitored Nrx1 $\beta$ and Nlg1 dynamics by uPAINT. NMDA caused a specific and time-dependent decrease in BFP-Nrx1 $\beta$ and AP-Nlg1 molecules from the cell surface (Fig. 5g,h), whereas their number remained constant under control conditions. Such a loss of the Nrx $1 \beta / \mathrm{Nlg} 1$ complex thus represents coordinated, activitydependent destabilization of trans-synaptic contacts at the singlemolecule level.

Differential dynamics of Nlg1 and LRRTM2 at synapses. To gain insight into the membrane dynamics of other synaptogenic proteins, we examined the behaviour of another excitatory postsynaptic adhesion molecule, LRRTM2, that competes with Nlg1 for the binding to presynaptic neurexins ${ }^{41-43}$. We first used the same dual-colour uPAINT analysis described above to simultaneously image AP-LRRTM2 in dendrites and BFP-Nrx1 $\beta$ in axons. Similarly to Nlg1, LRRTM2 was highly concentrated at synapses, where it formed a small number of confinement domains of $118 \pm 4 \mathrm{~nm}$ diameter overlapping with BFP-Nrx1 $\beta$ (Supplementary Fig. 10). However, the average diffusion coefficient of LRRTM2 was significantly lower than that of Nlg1, reflecting the fact that most LRRTM2 molecules (80\%) were localized at synapses, while very few (20\%) diffused freely on dendritic shafts (Fig. 6a-c). For comparison, the synaptic and dendritic fractions of $\mathrm{Nlg} 1$ were $60 \%$ and $40 \%$, respectively.

To confirm that Nlg1 and LRRTM2 also exhibited differential dynamics at the population level, we performed FRAP experiments. We labelled AP-Nlg1 and AP-LRRTM2 at the cell surface with $100 \mathrm{nM}$ mSA-Atto594 and photobleached individual synapses using a focused 561-nm laser beam. Fluorescence recovery was analysed for up to $30 \mathrm{~min}$ (Fig. 6d,e). Control FRAP experiments on Nlg1-GFP showed similar recovery curves (Supplementary Fig. 11). The recovery curves were fitted with a diffusion-reaction model described previously, which includes three adjustable parameters: the fraction of adhesion molecules trapped at the synapse, their steady-state exchange rate and the diffusion coefficient of free molecules ${ }^{44}$. We used the fastest AP-Nlg1 and LRRTM2 observed in uPAINT to estimate the diffusion coefficient of free molecules $\left(\sim 0.1 \mu \mathrm{m}^{2} \mathrm{~s}^{-1}\right)$ and adjusted the two other parameters to obtain the best fit. The exchange rate was approximately threefold lower for AP-LRRTM2 than for AP-Nlg1, and the trapped fraction was higher ( $81 \%$ versus $73 \%$, respectively). Therefore, FRAP predicts a longer synaptic residence time for LRRTM2 than for Nlg1, consistent with the uPAINT data.

Differential nanoscale organization of Nlg1 and LRRTM2. Finally, to gain insight on the nanoscale organization of Nlg1 and LRRTM2, we imaged Nlg1 and LRRTM2 by dSTORM in mature neurons (Fig. 7a-d). Unlike in uPAINT, originally diffusing molecules appear with a similar intensity to confined ones because of fixation, thus contributing to a homogenous signal in the shaft and spines. This effect was especially prominent for Nlg1, which was more diffusive than LRRTM2, resulting in a lower synaptic enrichment when compared with shaft levels $(2.7 \pm 0.6$ versus $5.3 \pm 0.8$, respectively). Furthermore, the dense labelling allows the identification of many simultaneous Nlg1-trapping events in the dendritic spine, yielding a fairly disperse localization with respect to the synapse centroid (Fig. 7e), but occasionally forming one or two clusters of similar sizes as those observed with uPAINT (average diameter $98 \pm 6 \mathrm{~nm}$; Fig. 7f,g). In contrast, LRRTM2 seemed to visit a more restricted postsynaptic area, mostly forming one compact mass comprising several small subclusters $(107 \pm 7 \mathrm{~nm})$ and being localized at a shorter distance from the centroid (Fig. 7b,d-g). This specific scattering of Nlg1 was not due to an increase in postsynaptic size compared with LRRTM2 (Fig. 7h). In summary, the two Nrx partners, Nlg1 and LRRTM2, differed considerably in their postsynaptic nano-organization and dynamics.

Finally, to rule out the possibility of a localization artefact due to AP-Nlg1 overexpression, saturating synaptic binding sites, we designed a rescue strategy by replacing endogenous Nlg1 with similar levels of exogenous AP-Nlg1. To this aim, we either co-expressed a short hairpin RNA to Nlg1 together with a resistant AP-Nlg1 construct in rat cultures, or expressed AP-Nlg1 in primary hippocampal cultures from Nlg1 KO mice. Western blots and Nrx1 $\beta$-binding assays confirmed that exogenous AP-Nlg1 levels roughly reached those of endogenous Nlg1 in both approaches (Supplementary Fig. 4). STORM images performed with mSA-Alexa647 on these cultures revealed a very similar distribution of rescue AP-Nlg1 compared with what we had previously observed using AP-Nlg1 expression alone, with a 
a

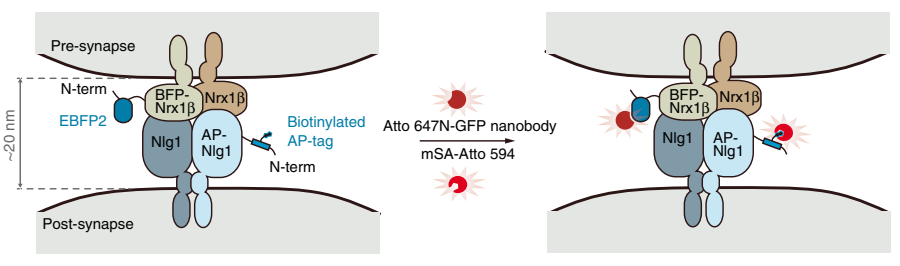

- Synaptic

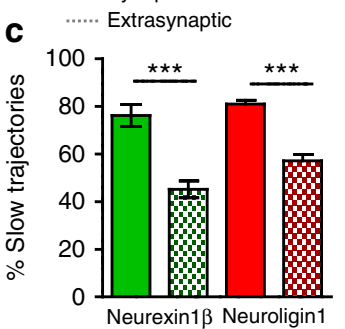

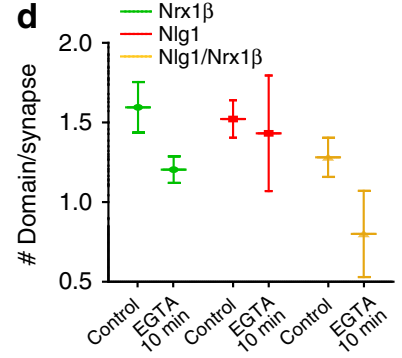

b
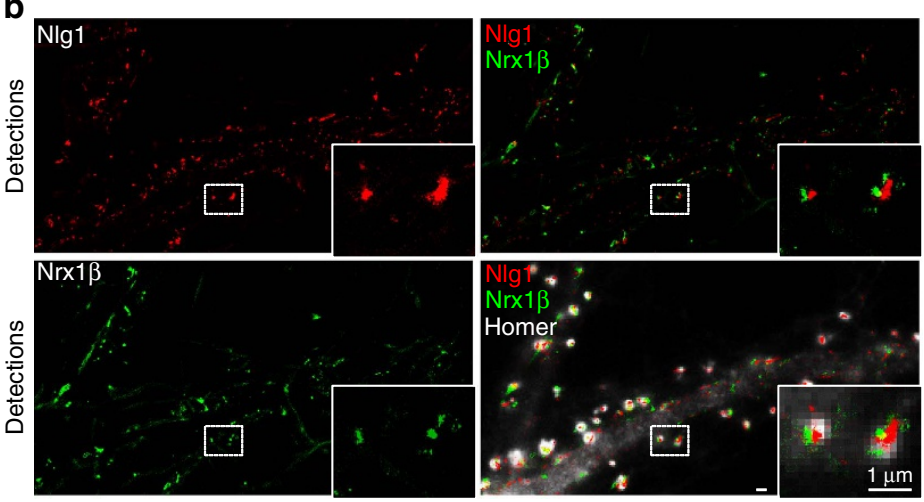

g

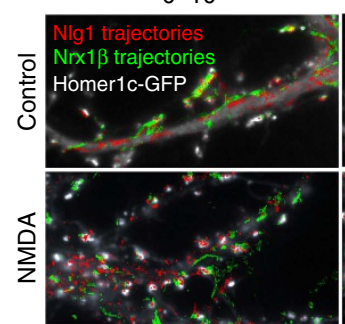

10-20'

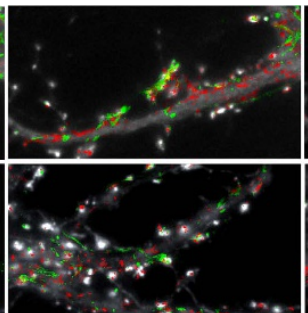

20-30'

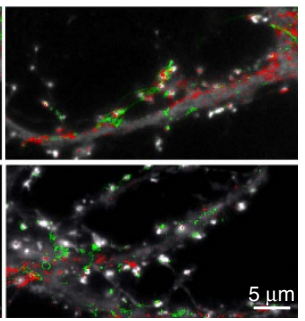

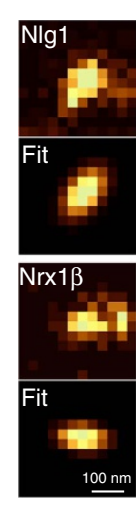

f

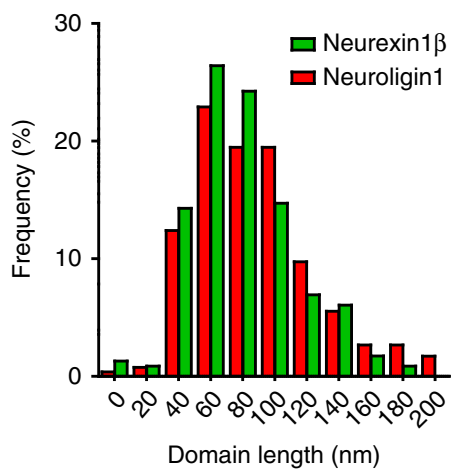

h

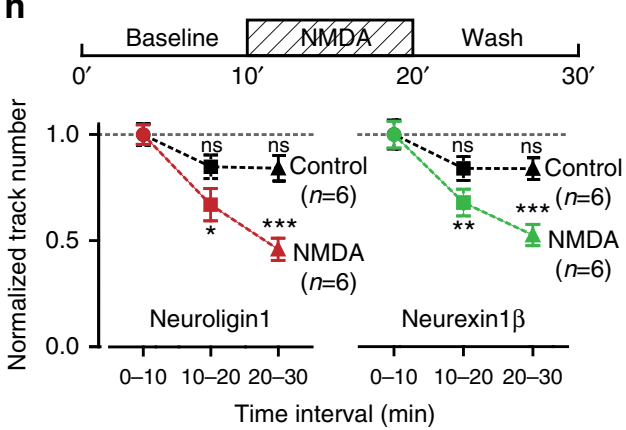

Figure 5 | Dual-colour super-resolution imaging of trans-synaptic contacts between neurexin-1/ and neuroligin-1. Neurons co-expressing AP-Nlg1, Bir ${ }^{E R}$ and Homer1c-GFP or expressing BFP-Nrx1 $\beta$ were co-cultured for 15 days and labelled with mSA-Atto 594 and Atto 647N nanobody. (a) Schematics of labelled adhesion molecules at the synapse. (b) Integrated density of Nlg1 (red) and Nrx1 (green) molecules at axon/dendrite contacts, identified from Homer1c-GFP signal (grey). (c) Percentages of slow trajectories for Nlg1 (red) and Nrx1 $\beta$ (green) were measured inside (solid) and outside (stippled) synapses (Nrx1 $\beta, n=12 ; \mathrm{Nlg1}, n=10$ cells from three different experiments $\left.{ }^{\star \star \star} P<0.0001\right)$. (d) Number of Nrx1 $($ green), Nlg1 (red) and apposed $\mathrm{Nrx1 \beta /NIg1} \mathrm{(yellow)} \mathrm{synaptic} \mathrm{clusters} \mathrm{before} \mathrm{and} \mathrm{after} 10$ min EGTA treatment ( $n=6$ cells for each condition from two different experiments).

(e) Examples of synaptic Nlg1 and Nrx1 $\beta$ clusters and corresponding two-dimensional anisotropic Gaussian fits. (f) Frequency distributions of Nlg1 and Nrx1 $\beta$ cluster lengths (NIg1: median 80.44, interquartile range (IQR) 56-102 nm, 531 clusters from 14 cells; Nrx1ß: median 75.17, IQR of 62-109 nm, 235 clusters from 11 cells. Data are from three different experiments). (g) Destabilization of trans-synaptic Nrx13/Nlg1 contacts by NMDA application (20 $\mu$ M, $10 \mathrm{~min}$ ). (h) Number of $\mathrm{Nlg} 1$ and Nrx1 $\beta$ trajectories over time on a 10-min NMDA treatment (red, green) or in control condition (black). The trajectory counts were normalized by their respective numbers before treatment $\left(n=6\right.$ cells for each condition from three different experiments ${ }^{\star \star \star} P<0.0001$, oneway analysis of variance).

similar spine/shaft enrichment (Fig. 7i-k). Together with the STORM images obtained in organotypic hippocampal slices from Nlg1 KO mice (Fig. 3e,f), these results demonstrate that the relative scattering of AP-Nlg1 within spines most likely reflects the localization of endogenous Nlg1.

\section{Discussion}

In this study, we developed and extensively characterized a new labelling method compatible with SRI, based on fluorescent monomeric streptavidin ( $\mathrm{mSA}$ ), to target recombinant biotinylated proteins at the cell membrane. mSA can be produced using standard bacterial protein production, and conjugated to fluorophores of interest with established coupling protocols.
This enables customization of the labelling toolbox with addition of new fluorophores, such as the recently reported siliconrhodamine derivatives that have improved photostability and fluorogenic properties. The high thermodynamic and aqueous stability of mSA allows for its conjugation to a variety of organic dyes with different physical properties (Atto-565, -594, -647N and Alexa 647) without having an impact on its behaviour in cellular environments, even when some dyes, for example, Atto $647 \mathrm{~N}$, are prone to incorporation in the membrane ${ }^{45}$. Although mSA cannot be used to label endogenous proteins, the low abundance of naturally biotinylated proteins ${ }^{46}$ allowed highly specific labelling of target proteins with all mSA conjugates tested. Therefore, using a similar labelling 
a
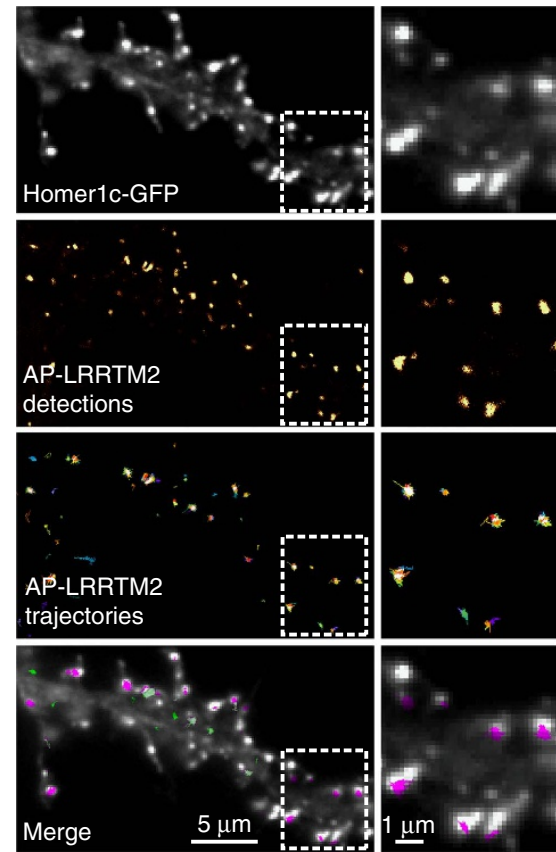

d
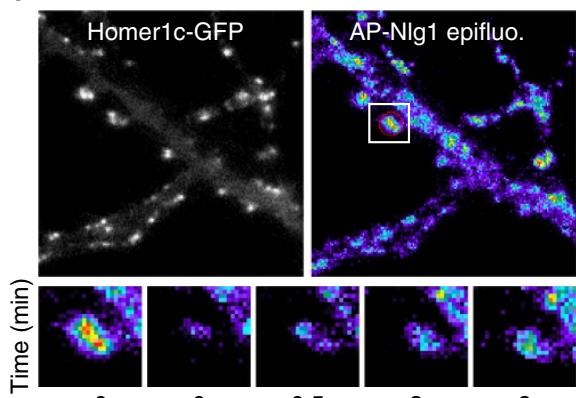

$<0$
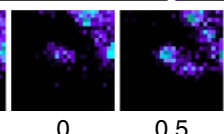

0.5

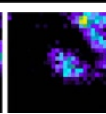

3

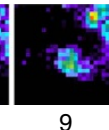

e

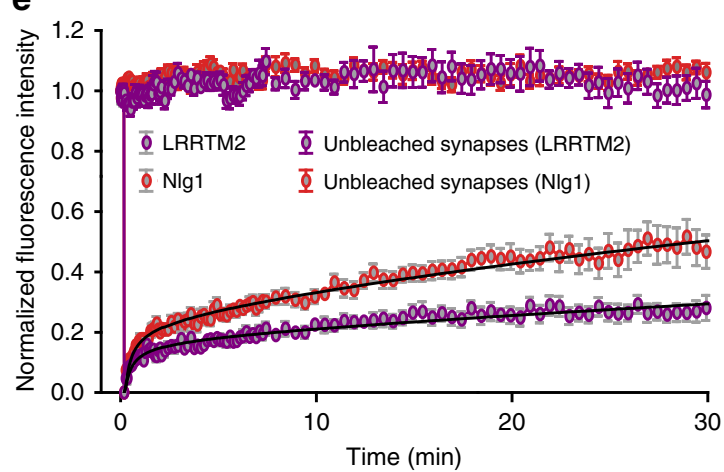

b

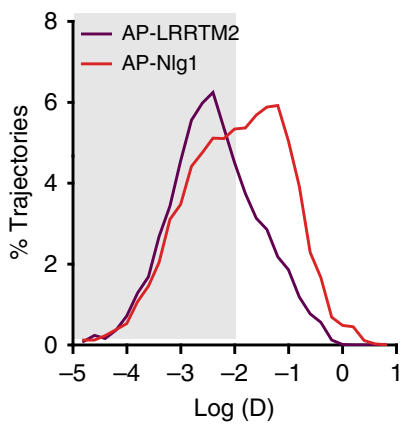

C

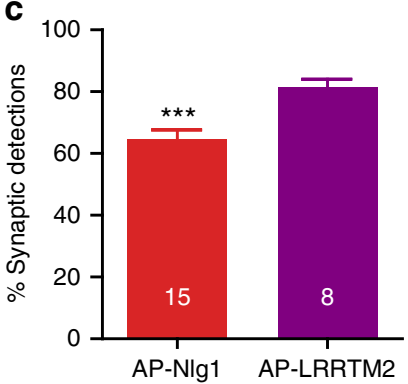

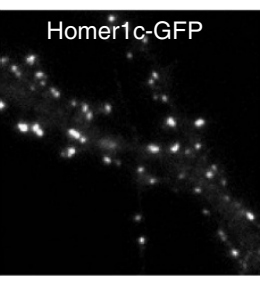
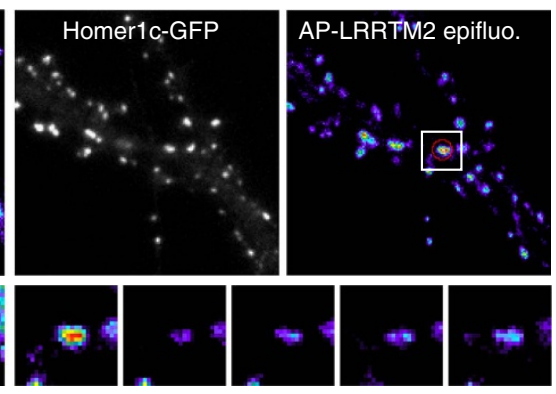

0.5

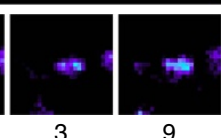

$<0$

9

Figure 6 | Comparison of NIg1 and LRRTM2 dynamics in mature hippocampal neurons. (a) DIV 15 neurons expressing AP-LRRTM2, Homer1c-GFP and BirA ${ }^{E R}$ were labelled using mSA-Atto 594 for UPAINT imaging of single LRRTM2 molecules. Super-resolved localization and trajectory maps are shown (green, fast-diffusing molecules, magenta, slow-moving molecules). Note the absence of diffusion on dendritic shafts. (b) Semi-log distribution of AP-LRRTM2 and AP-Nlg1 diffusion coefficients. (c) Corresponding percentage of synaptic LRRTM2 and NIg1 detections by uPAINT (LRRTM2, $n=8$; NIg1, $n=15$ cells from three different experiments). (d) FRAP experiments performed on AP-Nlg1 and AP-LRRTM2 labelled with mSA-Atto594.

(e) Corresponding normalized fluorescence recovery curves. The intensity of unbleached synapses is shown as control for observational photobleaching. Solid lines represent fits of the mean data points with the diffusion-reaction equation given in the Methods. The parameters obtained for Nlg1 and LRRTM2 were the fraction of free molecules $\phi=0.27$ and 0.19 and the turnover rate of adhesions $k_{\text {reac }}=1.4 \times 10^{-2}$ and $5.0 \times 10^{-3}$ min ${ }^{-1}$, respectively. The ratio of all synaptic molecules versus free molecules $(1 / \phi)$ gives 3.5 for Nlg1 and 5.1 for LRRTM2, closely corresponding to the synaptic enrichment values measured by dSTORM (NIg1, $n=25$; LRRTM2, $n=18$; NIg1 unbleached, $n=10$; LRRTM2 unbleached, $n=8$ cells for each condition from three different experiments). 
a
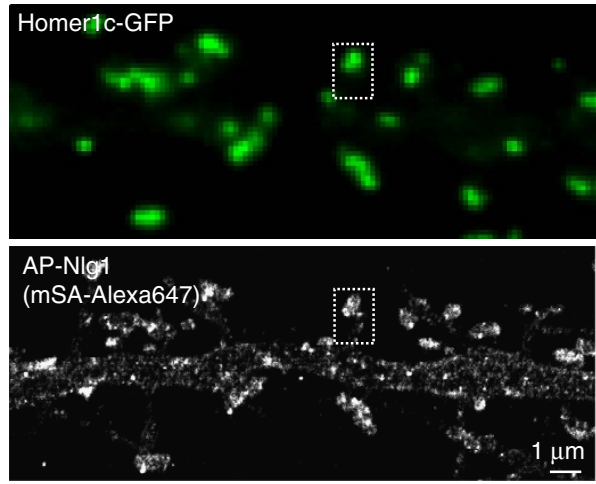

C
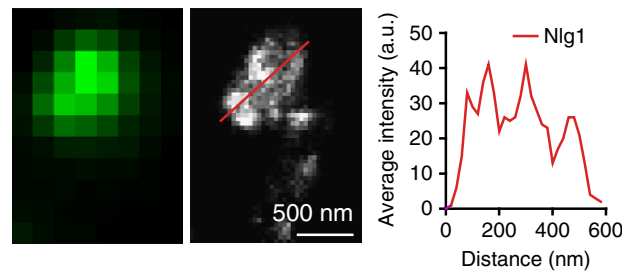

e

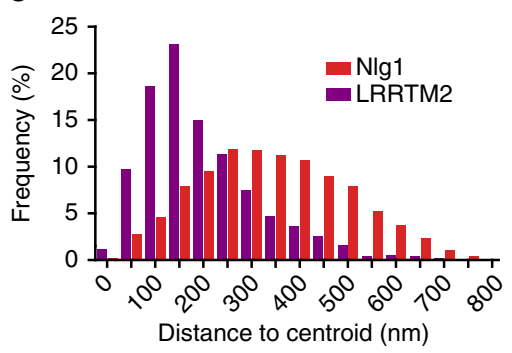

f

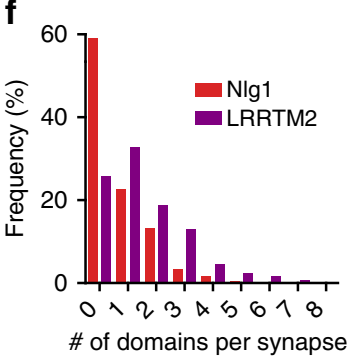

b

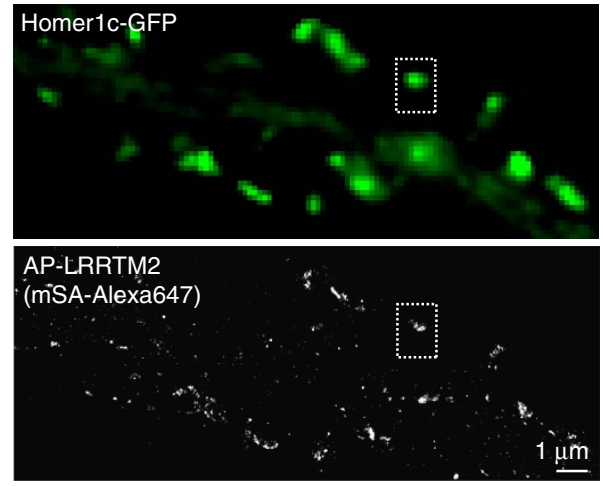

d

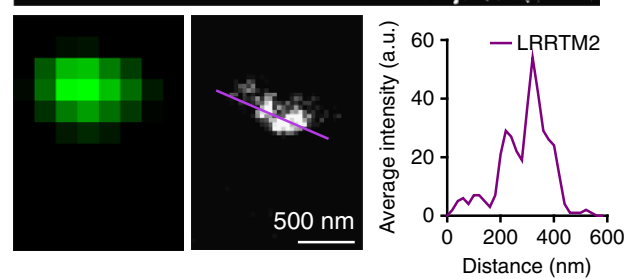

i

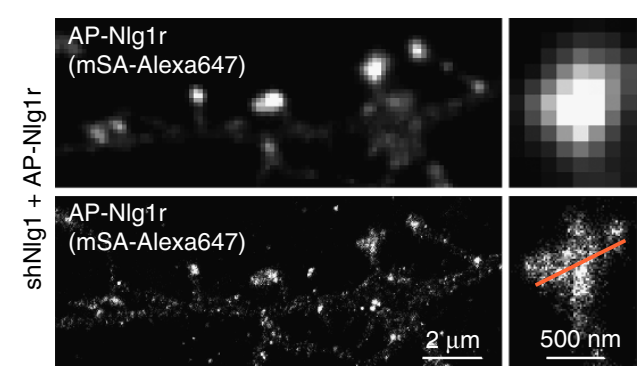

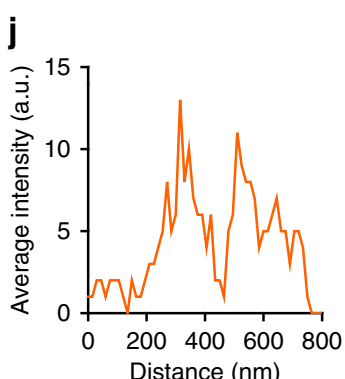

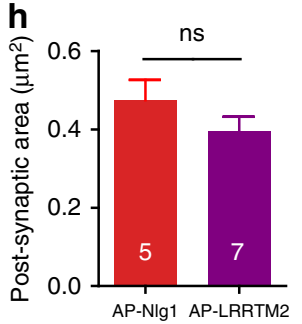

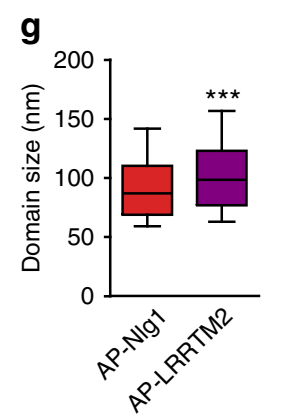

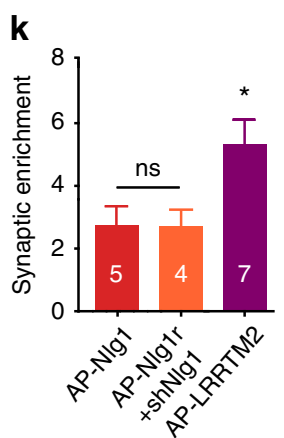

Figure 7 | Comparison of NIg1 and LRRTM2 organization in mature hippocampal neurons. (a,b) DIV 15 neurons expressing either AP-NIg1 or APLRRTM2, plus Homer1c-GFP and BirA ${ }^{E R}$ were labelled at high density with mSA-Alexa 647 for dSTORM imaging. Integrated densities over 40,000 frames are shown. (c,d) AP-Nlg1 and LRRTM2 fluorescence across linescans represented in $\mathbf{a}, \mathbf{b}$ insets show local fluorescence accumulation within synapses. (e) Dispersion of AP-NIg1 and AP-LRRTM2 molecules within synapses represented as the distribution of distances from individual synaptic detections relatively to the centroid of the Homer1c-GFP signal (LRRTM2, $n=7 ; \mathrm{Nlg1} n=5$ ). (f) Distribution of the number of locally enriched AP-NIg1 and AP-

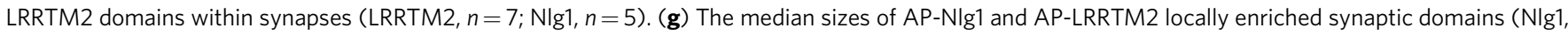
87.00, IQR 68-110, $n=5$; LRRTM2, 98.35, IQR 76-122, $n=7$; ${ }^{\star \star \star} P<0.0001$ ). (h) Synaptic area in DIV 15 neurons electroporated with AP-NIg1 or APLRRTM2 based on the Homer1c-GFP signal. (i) Representative STORM image of AP-Nlg1r expressed on a knockdown background in DIV 15 rat neurons, shown with the corresponding low-resolution mSA-Atto647 labelling. (j) Average intensity corresponding to the linescan in i showing local AP-Nlg1r fluorescence accumulation within a spine in a knockdown background, similar to AP-Nlg1. (k) Synaptic enrichment of AP-Nlg1, AP-Nlg1r co-expressed on a knockdown background and AP-LRRTM2 with respect to shaft levels (AP-Nlg1, $2.73 \pm 0.62, n=5 ;$ AP-Nlg1r, $2.54 \pm 0.34, n=4 ;$ AP-LRRTM2, 5.29 \pm 0.81 $\left.n=7 ;{ }^{\star} P<0.05\right)$. Data are from three different experiments for AP-Nlg1 and AP-LRRTM2, and two experiments for AP-Nlg1r.

scheme, we were able to explore real-time single-molecule dynamics at the nanoscale level (uPAINT), and to map representative protein organization at a precise time point to measure local densities within immobile structures (STED and dSTORM).
Antibodies are commonly used for labelling; however, highquality antibodies compatible with live imaging are not always available, a critical issue for the synaptic adhesion molecules studied here. Furthermore, our comparison of monomeric (mSA), dimeric (antibody) and tetrameric (streptavidin) probes clearly 
shows that, for live SRI applications, probe multivalence affects the measurements by generating artificial nanoscale clusters and biasing protein diffusion. For single-particle-tracking applications, mSA-Atto conjugates represent a significant improvement over large and multivalent nanoparticles such as antibody- or streptavidin-coated quantum dots, which often get sequestered at the periphery of the postsynaptic density because of steric hindrance $e^{31,32,47}$. However, because of their resistance to photobleaching compared with organic fluorophores, nanoparticles will remain a method of choice to track molecules for long durations, even more so with the recently developed non-blinking fluorescent nanoparticles ${ }^{48,49}$. mSA labelling is in many regards comparable to that of anti-GFP nanobody labelling, an example of monovalent detection recently used in SRI ${ }^{13}$. In some cases, however, labelling with mSA may be advantageous if the use of GFP as an antigenic tag is problematic. Indeed, the 15-amino-acid AP tag, which is similar in size to other commonly used epitopes, such as FLAG or c-Myc, can be readily inserted between structural domains or within protein loops with minimal perturbation of the native function. We demonstrated this point by fusing the AP tag on a short extracellular loop of stargazin that was previously targeted with an engineered $\mathrm{HA} \mathrm{tag}{ }^{36}$, whereas FP insertion at this location resulted in a dramatic mislocalization of the resulting fusion construct.

A benefit of developing an orthogonal technique of comparable capability includes the potential for dual-colour SRI. To this end, we simultaneously applied mSA and GFP nanobody labelling of trans-synaptic contacts in hippocampal neurons to reveal a differential dynamic organization of the two main $\operatorname{Nrx} 1 \beta$ adhesion partners at excitatory synapses, namely $\mathrm{Nlg} 1$ and LRRTM2. We showed a diffusional trapping of presynaptic Nrx1 $\beta$ and postsynaptic Nlg1, resulting in selective trans-synaptic apposition of those proteins at axon-dendrite contacts. Labelling of $\mathrm{Nrx} 1 \beta / \mathrm{Nlg} 1$ complexes was rapid with both $\mathrm{mSA}$ and GFP nanobody, neither of which affected their intrinsic interaction. In contrast, some labelling strategies, such as GFP complementation, have intrinsically slower kinetics owing to GFP folding and can artificially increase the binding strength between $\mathrm{Nrx}$ and $\mathrm{Nlg}$, thus enhancing synaptogenesis ${ }^{38}$. That Nrx1 $\beta$ escaped synapses more readily than Nlg1 on EGTA treatment suggests that the molecule may be retained at presynapses through extracellular calcium-dependent interactions, for example, with Nlgs and LRRTMs. This observation is consistent with the report that the Nrx intracellular domain is dispensable for Nlg1-induced presynaptic differentiation ${ }^{50}$. Nlg1 may be anchored at the synapses not only via extracellular interactions with Nrxs but also via intracellular interactions with PSD-95, which may be promoted by Nrx1 $\beta$ binding ${ }^{32}$.

Whether adhesion molecules are functionally regulated by synaptic activity remains an important, unresolved issue. Nlg1 interacts directly with the extracellular domain of NMDA receptors $^{51}$ and Nlg1 knockdown affects NMDAR-mediated synaptic transmission ${ }^{52,53}$. In addition, the synaptogenic effect induced by Nlg1 expression is abolished by chronic blockade of NMDA receptor activity ${ }^{53}$, whereas synapse elimination caused by the absence of Nlgs and LRRTMs is promoted by calcium-calmodulin kinase II (CamKII)-dependent synaptic activity $^{54}$. Our results show that both $\mathrm{Nlg} 1$ and $\mathrm{Nrx} 1 \beta$ trans-synaptic clusters rapidly vanish on NMDA treatment, suggesting that Nlg1 plays a role in activity-dependent synaptic remodelling through NMDA receptor activity. Proteolytic shedding of Nlg1 at the cell membrane by the metalloprotease MMP9 (ref. 55) or altered export of Nlg1 through phosphorylation by CamKII (ref. 56) provide possible mechanisms. In contrast to Nlg1, LRRTM2 is less mobile and is confined in more compact synaptic domains, suggesting that the molecule plays a strong role in maintaining trans-synaptic connectivity. This specific function of LRRTM2 might be achieved through direct extracellular interactions with presynaptic Nrxs or with postsynaptic AMPA receptors ${ }^{43}$, the latter playing a retrograde role on presynaptic differentiation ${ }^{57}$. Indeed, alternative splicing of presynaptic Nrx3 decreases both postsynaptic LRRTM2 and AMPA receptor levels ${ }^{58}$, and LRRTM knockdown alters AMPA receptor-mediated synaptic transmission and plasticity ${ }^{52}$. Both the number and size of synaptic LRRTM2 clusters match the recently identified AMPA receptor nanodomains ${ }^{59}$, suggesting that they may be structurally and/or functionally related. One interesting mechanism could be that extrasynaptic surface-diffusing AMPA receptors get dynamically trapped at Nrx1 $/$ Nlg1 adhesions through PSD-95 scaffolds $^{31,33}$, and then become stabilized at synapses by more durable interactions with LRRTM2.

Obtaining a more integrated view of the molecular ultrastructure of the synapse requires multicolour SRI of adhesion molecules, scaffolding proteins and neurotransmitter receptors. The penetrability and labelling efficiency reached with mSA, together with targeted expression of adhesion proteins such as $\mathrm{Nrx}$ and $\mathrm{Nlg}$ in intact organisms ${ }^{60}$ and improved optical detection in deep tissue ${ }^{61}$, should enable further investigation of activity-dependent modulation of synaptic protein organization with high spatial and temporal resolution. In addition to addressing specific questions in neuroscience, the mSA-based labelling strategy constitutes a significant progress towards developing a robust staining technique for fluorescence-based super-resolution microscopy, which should stimulate its application to a wide range of questions in fundamental biology.

\section{Methods}

DNA plasmids. The AP-Nlg1, AP-Nrx1 $\beta$, pDisplay-HA-6His-AP-CFP and BirA ${ }^{\mathrm{ER}}$ constructs $^{21,23}$ were kind gifts from A. Ting (MIT, Boston). HA-Nlg1 and short hairpin RNA to Nlg1 (shNlg1) were obtained from P. Scheiffele (Biozentrum, Basel) The AP-Nlg1 rescue was generated by changing the nucleotide sequence of AP-Nlg1 recognized by the shNlg1 $5^{\prime}$-gaaggtactggaaatctg- $3^{\prime}$ to $5^{\prime}$-gaGggCacGggTaaCTtg- $3^{\prime}$, using silent mutations. Myc-LRRTM2 (ref. 43) was a gift from J. de Wit (Leuwen, Belgium). Nlg1 with GFP insertion at position 728aa (Nlg1-GFP) was generously provided by T. Dresbach (Goettingen, Germany) ${ }^{62}$. AP-LRRTM2 was generated using the In-Fusion HD Cloning kit (Clontech), replacing the myc-tag from myc-LRRTM2 by the AP Tag (amino-acid sequence GLNDIFEAQKIEWHE). The AP tag sequence was amplified from pDisplay-HA-6His-AP-CFP. Oligonucleotides used were as follows: LRRTM2-1F, 5' -ACTAGTTGTCCACCCAAATG-3';

LRRTM2-2R, 5' -GCTAGCCGCCATACCCAG-3'; AP-5F, 5'-GGTATGGCGGC TAGCggcctgaacgatatcttcg-3'; AP-6R, 5'-GGGTGGACAACTAGTctcgtgccactc gatctt- $3^{\prime}$. HomerlcGFP was a gift from S. Okabe (Tokyo University, Japan). BFPNrx1 $\beta$ was derived from human GFP-Nrx1 $\beta$ (gift from M. Missler, Münster University, Germany) by replacing the GFP sequence by PCR-amplified-EBFP2 from pEBFP2-Nuc (obtained from Addgene, plasmid 14893). AP-SEP-transferrin receptor was a kind gift from D. Perrais (IINS Bordeaux). HA-stargazin ${ }^{36}$ was a gift from $\mathrm{R}$ Nicoll (UCSF, USA). AP-stargazin was obtained by inserting a synthetic fragment containing the AP tag between residues 50 and 51 (in the first extracellular loop, same position as HA-stargazin) using HindIII and BspEI restriction sites. For comparison of the impact of FP insertion, mCherry was inserted into the same stargazin construct either at the same position as the AP tag (first extracellular loop, between residues 50 and 51, AgeI and NheI restriction sites) or at position - 56 (BgIII site) with respect to the C terminus (cytoplasmic tail). The plasmid for bacterial expression of the anti-GFP nanobody ${ }^{14}$ was a kind gift from Alexis Gautreau (LEBS, Gif-sur-Yvette, France). mSA was subcloned from the previously described pRSET-A vector ${ }^{25,26}$ into pET-IG, a homemade vector derived from pET-24 (Novagen) and engineered to incorporate after the start codon a decahistidine tag immediately followed by a Tobacco Etch Virus cleavage site (-ENLYFQS-) and no tag on the C terminus.

Protein expression and purification. Nrx1 $\beta$-Fc was obtained as follows. Conditioned medium from a stable hygromycin-resistant HEK cell line producing Nrx1 $\beta$-Fc lacking splice insert 4 was collected, and recombinant Nrx1 $\beta$-Fc was purified on a protein G column (HiTrap Protein G HP, GE Healthcare) to a concentration of $0.6-1.0 \mathrm{mg} \mathrm{ml}^{-1}$ (refs 31,32 ). mSA was produced similarly to previously reported methods ${ }^{25,26}$, with slight modifications. Briefly, pET-IG-mSA was transformed into E. Coli BL21 codon plus (DE3)-RIL and expressed for $12 \mathrm{~h}$ at 
$16^{\circ} \mathrm{C}$ in $300 \mathrm{ml}$ using an autoinduction protocol ${ }^{63}$. The cells were harvested and resuspended in $9 \% \mathrm{NaCl}$, transferred into a conical tube and kept at $-80^{\circ} \mathrm{C}$ until purification. The cell pellet was thawed and resuspended in $40 \mathrm{ml}$ of freshly prepared denaturing lysis buffer $(50 \mathrm{mM}$ Tris acetate, $\mathrm{pH} 8.0,8 \mathrm{M}$ urea and $40 \mu$ l Protease Cocktail III from Calbiochem) and gently mixed for $30 \mathrm{~min}$ at $4{ }^{\circ} \mathrm{C}$. Cells were lysed by sonication and lysates were cleared by centrifugation at $10,000 \mathrm{~g}\left(60 \mathrm{~min}, 4^{\circ} \mathrm{C}\right)$. HIS-Buster Cobalt Affinity gel (AMOCOL, $1 \mathrm{ml}$ ) was added and incubated with the supernatant for $2 \mathrm{~h}$ at $4{ }^{\circ} \mathrm{C}$. The resin was collected, washed extensively with washing buffer $(50 \mathrm{mM}$ TrisOAc, $\mathrm{pH} 8.0,300 \mathrm{mM} \mathrm{NaCl}, 5 \mathrm{mM}$ imidazole and $8 \mathrm{M}$ urea, freshly prepared) and the protein was eluted with $3 \times 1 \mathrm{ml}$ of elution buffer $(50 \mathrm{mM}$ TrisOAc, $\mathrm{pH} 8.0,300 \mathrm{mM} \mathrm{NaCl}, 500 \mathrm{mM}$ imidazole, $8 \mathrm{M}$ urea, freshly prepared). The elution fractions were added drop by drop to $40 \mathrm{ml}$ of ice-cold PBS buffer ( $5 \mathrm{mM} \mathrm{Na}_{2} \mathrm{HPO}_{4}, 5 \mathrm{mM} \mathrm{NaH}_{2} \mathrm{PO}_{4}$ and $150 \mathrm{mM} \mathrm{NaCl}$ ) containing $0.3 \mathrm{mg} \mathrm{ml}^{-1}$ d-biotin, $0.2 \mathrm{mg} \mathrm{ml}^{-1}$ oxidized glutathione and $1 \mathrm{mg} \mathrm{ml}^{-1}$ reduced glutathione under rapid stirring to refold the protein. The precipitates were removed by centrifugation. The refolded protein was concentrated to $\sim 1 \mathrm{mg} \mathrm{ml}^{-1}$ using Amicon Ultra centrifugal filters with a $10-\mathrm{kDa}$ cutoff. The anti-GFP nanobody was expressed in the same conditions as mSA and purified under native conditions by affinity chromatography using the HIS-Buster Nickel Affinity gel. The protein was dialysed in PBS buffer and concentrated to $\sim 1 \mathrm{mg} \mathrm{ml}^{-1}$ using Amicon Ultra centrifugal filters with a 10-kDa cutoff. mSA and anti-GFP nanobody were kept at $4^{\circ} \mathrm{C}$ and used for coupling to fluorophores within $24 \mathrm{~h}$. The modified, negatively charged biotin (Biot-DDDY-COOH) to reduce membrane permeability ${ }^{27}$ was obtained by manual standard Fmoc-based solid-phase peptide synthesis.

Protein and antibody coupling to fluorophores. All proteins (mSA, anti-GFP nanobody, streptavidin (85878, Sigma-Aldrich), mouse monoclonal anti-biotin (033700 , Invitrogen)) were prepared in PBS at $\sim 1 \mathrm{mg} \mathrm{ml}^{-1}$. Coupling to Atto $647 \mathrm{~N}$, Atto 594 and Alexa 647 was performed following the recommended procedures from the manufacturers (ATTO-TEC and Life Technologies) with the corresponding NHS ester derivatives of each dye. Briefly, labelling was conducted in the dark at room temperature for $1 \mathrm{~h}$. Excess dye was removed using Sephadex G-25 medium (PD MiniTrap G-25, GE Healthcare) by elution with PBS. mSA-Atto 647N, mSA-Atto 594, mSA-Alexa 647 and the Atto 647N-GFP nanobody were further purified to homogeneity by size exclusion chromatography with a Superdex 75 HiLoad 16/60 column (GE Healthcare) on an AKTA purifier system (GE Healthcare) using PBS as a running buffer. Labelled proteins were concentrated to $\sim 0.2 \mathrm{mg} \mathrm{ml}^{-1}$ using Amicon Ultra centrifugal filters with a $10-\mathrm{kDa}$ cutoff. All proteins were aliquoted and flash-frozen for storage at $-80^{\circ} \mathrm{C}$ until use.

Cell culture and electroporation. Gestant rat females were purchased weekly (Janvier Labs, Saint-Berthevin, France), while wild-type and Nlg1 KO mouse strains obtained from F. Varoqueaux and N. Brose (MPI Goettingen) were raised in our animal facility. Animals were handled and killed according to European ethical rules. Dissociated hippocampal neurons from E18 Sprague-Dawley rat embryos or P0 mice (from Nlg1 WT or KO background) were prepared as described ${ }^{64}$, and electroporated with the Amaxa system (Lonza) using 500,000 cells per cuvette. The following plasmid combinations were used: (GFP or Homer1c-GFP) $+\mathrm{BirA}^{\mathrm{ER}}+$ (AP-Nlg1, AP-LRRTM2, AP-STG or AP-Nrx1 $\beta$ ) (1.5:1.5:1.5 $\mu \mathrm{g}$ DNA), BFP-Nrx1 $\beta$ or GFP-Nrx1 $\beta$ ( $4.5 \mu \mathrm{g}$ DNA), Nlg1-GFP ( $4 \mu \mathrm{g}$ DNA). Electroporated neurons were resuspended in Minimal Essential Medium supplemented with 10\% horse serum (MEM-HS) and plated on 18-mm coverslips previously coated with $1 \mathrm{mg} \mathrm{ml}^{-1}$ polylysine for $2 \mathrm{~h}$ at a concentration of 50,000 cells per coverslip. Three hours after plating, coverslips were flipped onto $60-\mathrm{mm}$ dishes containing a glial cell layer in Neurobasal medium (NB for rat cultures or NB-A for mouse cultures) supplemented with $2 \mathrm{mM}$ L-glutamine and $1 \times$ NeuroCult SM1 Neuronal supplement (STEMCELL Technologies) and cultured for 2 weeks at $37^{\circ} \mathrm{C}$ and $5 \%$ $\mathrm{CO}_{2}$. Astrocyte feeder layers were prepared from the same embryos, plated between 20,000 and 40,000 cells per 60-mm dish and cultured in MEM (Fisher Scientific, cat. no. 21090-022) containing $4.5 \mathrm{gl}^{-1}$ Glucose, $2 \mathrm{mM}$ L-glutamine and $10 \%$ horse serum (Invitrogen) for 14 days. For biochemistry experiments, electroporated neurons from rats or mice were seeded in a six-well plate coated with $1 \mathrm{mg} \mathrm{ml}^{-1}$ polylysine for $2 \mathrm{~h}$ at a concentration of 500,000 cells per well. Three hours after plating, the medium was replaced by conditioned Neurobasal medium (rat) or Neurobasal-A medium (mice), supplemented with $2 \mathrm{mM} \mathrm{L-glutamine}$ and $1 \times$ NeuroCult SM1 Neuronal supplement (STEMCELL Technologies) and renewed every 3-4 days. Ara-C (3.4 $\mu \mathrm{M})$ was added at DIV 3 and DIV 13. COS-7 and HEK-293 cells were cultured in DMEM (GIBCO/BRL) supplemented with $10 \%$ fetal bovine serum, 100 units $\mathrm{ml}^{-1}$ penicillin and $100 \mu \mathrm{g} \mathrm{ml}^{-1}$ streptomycin. Heterologous cells were electroporated with TfR-SEP-AP + BirA $^{\mathrm{ER}}$ (1.5:2 $\mu \mathrm{g}$ DNA for 2 million cells) or stargazin-mcherry ( $3.5 \mu \mathrm{g}$ DNA for 2 million cells) constructs with the Amaxa system (Lonza) using 500,000 cells per cuvette.

Neuronal lysates. DIV 14 mouse or rat neuronal cultures were rinsed in ice-cold PBS, and then scraped into $100 \mu$ RIPA buffer (50 mM HEPES, $10 \mathrm{mM}$ EDTA, $0.1 \%$ sodium dodecyl sulfate, $1 \%$ IGEPAL CA-630 and $0.5 \%$ sodium deoxycholate, $\mathrm{pH}=7.2$ ). Homogenates were kept $15 \mathrm{~min}$ on ice and centrifuged at $8,000 \mathrm{~g}$ for $15 \mathrm{~min}$ at $4^{\circ} \mathrm{C}$ to remove cell debris. Ten microlitres per condition were loaded for western blots.
SDS-PAGE and immunoblotting. Samples were separated by TGX stain-free precast gels (4-15\% gradient, Bio-Rad), ultraviolet-activated with ChemiDoc Touch system (Bio-Rad) for direct imaging of total proteins and then transferred to nitrocellulose membranes for immunoblotting analysis. After blocking with 5\% non-fat dried milk in Tris-buffered saline Tween-20 (TBST; $28 \mathrm{mM}$ Tris, $136.7 \mathrm{mM}$ $\mathrm{NaCl}, 0.05 \%$ Tween-20, $\mathrm{pH} 7.4$ ) for $45 \mathrm{~min}$ at room temperature, membranes were incubated with rabbit anti-Nlg1 (129013, Synaptic systems) diluted at 1:1,000 with $0.5 \%$ non-fat dried milk in TBST, followed by horse radish peroxidase-conjugated anti-rabbit antibody (Jackson ImmunoResearch) for $1 \mathrm{~h}$ at room temperature. Target proteins were detected by chemiluminescence with Super signal West Dura (Pierce) on the ChemiDoc Touch system (Bio-Rad). The theoretical molecular weight of Nlg1 is $93 \mathrm{kDa}$, but the apparent molecular weight in immunoblots is $\sim 130 \mathrm{kDa}$, likely due to glycosylations ${ }^{65}$. For quantification, the intensity of the chemiluminescence signal of each lane was normalized by the total protein signal on the same lane, revealed by the stain-free technology.

\section{Organotypic cultures and single-cell electroporation. Organotypic}

hippocampal slice cultures were prepared from either wild-type or Nlg1 KO mice (C57Bl6/J strain), as described below ${ }^{66}$. Briefly, animals at postnatal day 4-6 were quickly decapitated and their brains placed in ice-cold Gey's balanced salt solution under sterile conditions. Hippocampi were dissected out and coronal slices $(350 \mu \mathrm{m})$ were cut using a tissue chopper (Mcllwain) and incubated with serum-containing medium on Millicell culture inserts (CM, Millipore). The medium was replaced every 2-3 days. After 3-4 days in culture, CA1 pyramidal cells were processed for single-cell electroporation with plasmids encoding enhanced GFP (EGFP) along with AP-Nlg1 and Bir $\mathrm{A}^{\mathrm{ER}}$ in equal proportions. The pipette containing $33 \mathrm{ng} \mathrm{ll}^{-1}$ total DNA was placed close to the soma of individual CA1 pyramidal neurons. Electroporation was performed by applying three square pulses of negative voltage $(10 \mathrm{~V}, 20 \mathrm{~ms}$ duration) at $1 \mathrm{~Hz}$, and then the pipette was gently removed. Three to five neurons were electroporated per slice, and the slice was placed back in the incubator for several days before imaging.

Single Molecule Tracking (uPAINT). uPAINT experiments were carried out as previously reported ${ }^{8}$. Cells were mounted in Tyrode solution (15 mM D-glucose, $108 \mathrm{mM} \mathrm{NaCl}, 5 \mathrm{mM} \mathrm{KCl}, 2 \mathrm{mM} \mathrm{MgCl}, 2 \mathrm{mM} \mathrm{CaCl}_{2}$ and $25 \mathrm{mM}$ HEPES, $\mathrm{pH} 7.4$ ) containing $1 \%$ globulin-free BSA (Sigma) in an open Inox observation chamber (Life Imaging Services, Basel, Switzerland). The chamber was placed on an inverted microscope (Nikon Ti-E Eclipse) equipped with an EMCCD camera (Evolve, Roper Scientific, Evry, France), a thermostatic box (Life Imaging Services) providing air at $37^{\circ} \mathrm{C}$ and an apochromatic (APO) total internal reflection fluorescence (TIRF) $\times 100$ oil 1.49 numerical aperture (NA) objective. BFP- and GFP-expressing cells were detected using a mercury lamp (Nikon Xcite) and the following filter sets (SemROCK, USA): BFP (excitation: FF01-379/34; dichroic: FF-409Di03; emission: FF01-440/40); EGFP (excitation: FF01-472/30; dichroic: FF-562Di02; emission: FF01-593/40). Cells expressing the different AP constructs, or BFP- or GFP- Nrx1 $\beta$, were labelled using low concentrations of Atto 594-conjugated mSA, Atto 594conjugated streptavidin and Atto 594-anti-biotin, or Atto 647N- and 594-conjugated GFP nanobody $(1 \mathrm{nM})$ to isolate single molecules. The GFP nanobody recognizes BFP, given the high sequence homology with GFP. A four-colour laser bench (405; 488; 561; and $642 \mathrm{~nm}, 100 \mathrm{~mW}$ each; Roper Scientific) is connected through an optical fibre to the TIRF illumination arm of the microscope. Laser powers were controlled through acousto-optical tunable filters driven by the Metamorph software (Molecular Devices, USA). Atto 594 and Atto 647N were excited with the 561- and 642-nm laser lines through a four-band beam splitter (BS R405/488/561/635, SemRock). Samples were imaged by oblique laser illumination, allowing the excitation of individual Atto-conjugated ligands bound to the cell surface, without illuminating ligands in solution. Fluorescence was collected using FF01-617/73 and FF01-676/29 nm emission filters (SemRock), respectively, placed on a filter wheel (Suter). Stacks of 2,000-4,000 consecutive frames were obtained from each cell, with an integration time of 20-50 ms. Multicolour fluorescent 100-nm beads (Tetraspeck, Invitrogen) were used to register long-term acquisitions and correct for lateral drifts. In some experiments, EGTA $(10 \mathrm{mM})$ was applied directly in the bath and left for $10 \mathrm{~min}$. For NMDA experiments, a Gilson perfusion system was used to perfuse and change the medium up to $1 \mathrm{~h}$ during acquisitions. Neurons were perfused with Tyrode solution containing the fluorescent labels during the whole acquisition time Cells were recorded in control solution for $10 \mathrm{~min}$, treated with $20 \mu \mathrm{M}$ NMDA in Tyrode for $10 \mathrm{~min}$ and allowed to recover in control solution for $10 \mathrm{~min}$. Control cells were kept in Tyrode solution the whole time. Acquisitions were steered using the Metamorph software (Molecular Devices) in a streaming mode at $50 \mathrm{~Hz}$. Cycles of two consecutive series of acquisitions were made sequentially up to $1 \mathrm{~h}$. One series corresponds to GFP (20 frames, $50 \mathrm{~Hz}), \mathrm{mSA}$-Atto $594(1,500$ frames, $50 \mathrm{~Hz})$ and Atto $647 \mathrm{~N}$-GFP nanobody $(1,500$ frames, $50 \mathrm{~Hz})$.

Trajectory analysis and image reconstruction. Image stacks were analysed using a custom programme running on Metamorph based on wavelet segmentation for localization and simulated annealing algorithms for tracking, described earlier ${ }^{67,68}$. The programme allows both the reconstruction of the super-resolution image by summing the positions of localized single molecules into a single image, and tracking of localized molecules through successive images. The instantaneous 
diffusion coefficient, $D$, was calculated for each trajectory from linear fits of the first 4 points of the mean square displacement (MSD) function versus time. Slow trajectories were defined as trajectories with diffusion coefficients below $0.0093 \sim 0.01 \mu \mathrm{m}^{2} \mathrm{~s}^{-1}$ (Supplementary Fig. 4). This threshold corresponds to molecules exploring a region smaller than that defined by the spatial resolution of the system $(\sim 0.054 \mu \mathrm{m}$, full-width at half-maximum) during the time used to fit the initial slope of the MSD (4 points, $20 \mathrm{~ms}$ ) and is given by $D_{\text {threshold }}=(0.054 \mu \mathrm{m})^{2} /(4 \times 4 \times 0.02 \mathrm{~s}) \sim 0.0093 \mu \mathrm{m}^{2} \mathrm{~s}^{-1}$, as described earlier ${ }^{16}$. Spatial resolution was determined using fixed Atto samples. Overall, 415 two-dimensional (2D) distributions of single-molecule positions belonging to long trajectories ( $>10$ frames) were measured by bi-dimensional Gaussian fitting and the resolution determined as $2.3 \sigma_{x y}$, where $\sigma_{x y}$ is the pointing accuracy. For trajectory analysis, synapses were identified by wavelet-based image segmentation of the Homerlc-GFP postsynaptic marker. The corresponding binary masks were used to sort single-particle data analyses to specific synaptic regions. For NMDA experiments, each mSA and nanobody sequence was analysed separately as described, and data were pooled over 10-min intervals, to obtain representative trajectories of protein populations. The percentage of synapses containing Nlg1 was defined as the ratio between synapses containing at least 10 detections of AP-Nlg1 over the total number of synapses on super-resolved images obtained in the same conditions for mSA and biotin antibody. Synaptic coverage was determined from super-resolved detection maps as the ratio between threshold areas containing detections over a whole synaptic region determined from the low-resolution Homerlc signal. The percentage of synaptic detection is defined as the number of detections within synapses defined by Homerlc divided by the total number of detections. For dual-colour imaging of $\mathrm{Nlg} 1$ and $\mathrm{Nrx} 1 \beta$, areas with high signal density were identified as domains by wavelet segmentation. Their number and size were extracted from 2D isotropic Gaussian fitting, and their length was determined as the full-width at half-maximum. In the supplemental figures, the number of objects per frame was analysed using the 'Analyse Particles' plugin in ImageJ and averaged per stack of 2,000 frames acquired every $50 \mathrm{~ms}$, and the number of trajectories per $10 \mu \mathrm{m}$ was determined as the average number of trajectories per dendritic segments of $10 \mu \mathrm{m}$. MSD analysis was performed by filtering trajectories with $D<0.01$ or $D>0.01 \mu^{2} \mathrm{~s}^{-1}$ (Supplementary Fig. 4). The MSD curve as a function of time for $D<0.1 \mu \mathrm{m}^{2} \mathrm{~s}^{-1}$ was fitted by one-phase association curve on GraphPad to extract the confinement area, which was estimated to be $\sim 118 \mathrm{~nm}$.

dSTORM. Primary cultured neurons co-expressing Homerlc-GFP, BirA ${ }^{\text {ER }}$ and AP-Nlg1 or CA1 neurons in organotypic slice cultures from Nlg1 KO mice that were single-cell-electroporated with GFP, BirA ${ }^{\mathrm{ER}}$ and AP-Nlg1 were surface-labelled with a high concentration $(100 \mathrm{nM})$ of mSA-Alexa647, biotin antibody-Alexa647 or streptavidin-Alexa647 in Tyrode solution for $10 \mathrm{~min}$, and were rinsed and fixed with $4 \%$ PFA- $0.2 \%$ glutaraldehyde in PBS-BSA $1 \%$ for $10 \mathrm{~min}$ at room temperature or $2 \mathrm{~h}$ at $4{ }^{\circ} \mathrm{C}$. dSTORM imaging of cultured neurons and astigmatic-based 3D dSTORM imaging of organotypic brain slices on neurons located close to the coverslip surface was performed. We used an inverted motorized microscope (Nikon Ti, Japan) equipped with a $\times 1001.49 \mathrm{NA}$ PL-APO objective and a perfect focus system, allowing long acquisition in oblique illumination mode. Both the ensemble and single-molecule fluorescence were collected by using a quad-band dichroic filter (Di01-R405/488/561/635, Semrock). The fluorescence was collected using a sensitive EMCCD (Evolve, Photometrics, USA). For 3D imaging in organotypic brain slices, astigmatism was applied using an adaptive optics system (MicAO-Imagine Optic). 3D calibration was established using 100-nm fluorescent beads (Tetraspeck, Life Technologies) adhered to the slice surface. Single-molecule localization and reconstruction was performed online with automatic feedback control of the lasers using WaveTracer module, enabling optimal single-molecule density during the acquisition ${ }^{68}$. The acquisition and localization sequences were driven by MetaMorph (Molecular Devices) in a streaming mode at 50 frames per second (20-ms exposure time) using an area equal to or less than $256 \times 256$ pixel region of interest. The brain slice was mounted in an oxygen-scavenging imaging buffer ${ }^{6}$ and sealed between two glass coverslips. Images were composed of 1,038,506 localizations analysed from 64,000 frames, over which sample drift was corrected by localizing and tracking the displacement of cellular autofluorescence. Super-resolution reconstructions were generated with the VISP software ${ }^{69}$, and detection density maps were generated with a neighbourhood radius of $400 \mathrm{~nm}$. The resulting lateral resolution is $20 \mathrm{~nm}$ for primary neuronal cultures and $50 \mathrm{~nm}$ for slice cultures, while the axial resolution is $\sim 50 \mathrm{~nm}$. The number of clusters per $\mu \mathrm{m}^{2}$ was determined by wavelet segmentation based on areas with strong signal intensity compared with neighbouring areas ${ }^{67,68}$ on the super-resolved dSTORM images generated from 40,000 frames. Synaptic enrichment was defined as the ratio between the average number of synaptic detections (area given by the Homerlc-GFP signal) and the average number of extrasynaptic detections. The dispersion of Nlg1 and LRRTM2 molecules compared with Homer centroid was computed as the distribution of the distances between each detection event and the Homer centroid. Enrichment domains were defined by wavelet segmentation of areas with higher labelling densities relatively to neighbouring environment as illustrated by the linescans in Fig. 7, and their number per synapse was counted for LRRTM2 and Nlg1. Domain sizes were extracted from 2D isotropic Gaussian fittings as the average full-width at halfmaximum.
STED imaging and data analysis. $\mathrm{CA} 1$ neurons co-expressing EGFP, $\mathrm{Bir}^{\mathrm{ER}}$ and AP-Nlg1 in organotypic slices from wild-type mice were surface-labelled with high concentration of mSA-Atto647N $(100 \mathrm{mM})$ in Tyrode solution for $10 \mathrm{~min}$, rinsed, observed live using a commercial STED microscope (TCS SP5, Leica) and thermostated to $37^{\circ} \mathrm{C}$. STED illumination of Atto $647 \mathrm{~N}$ was performed using a 633-nm pulsed laser providing excitation, and a pulsed bi-photon laser (Mai Tai; Spectra-Physics) tuned to $765 \mathrm{~nm}$ and going through a $100-\mathrm{m}$ optical fibre to enlarge pulse width ( $100 \mathrm{ps}$ ) used for depletion. A doughnut-shaped laser beam was achieved through two lambda plates. Fluorescence light between 650 and $740 \mathrm{~nm}$ was collected using a photomultiplier, using a HCX PL-APO CS $\times 100 / 1.40 \mathrm{NA}$ oil objective and a pinhole open to one time the Airy disk $(60 \mu \mathrm{m})$. A fivefold zoomed area of $512 \times 512$ pixels, corresponding to a pixel size of $30 \mathrm{~nm}$, was scanned at $50 \mathrm{~Hz}$. In-depth acquisitions were performed by scanning hippocampal slices with an increment of $1 \mu \mathrm{m}$ in $z$ over $60 \mu \mathrm{m}$. When indicated, raw data were deconvoluted using the measured point spread function of the system and the Richardson-Lucy algorithm with Huygens Professional (Huygens Software). 3D reconstructed movies were made using Imaris (Bitplane).

FRAP experiments and analysis. Neurons electroporated with Homer1c-GFP, $\mathrm{BirA}^{\mathrm{ER}}$ and AP-Nlg1 or AP-LRRTM2 and cultured for 2 weeks were labelled with high concentration of mSA-Atto594 $(100 \mathrm{nM})$ for $10 \mathrm{~min}$, and then rinsed, mounted in Tyrode solution and observed under the same set-up used for uPAINT. The laser bench (comprising 488, 561 and $642 \mathrm{~nm}$ lasers, $100 \mathrm{~mW}$ each, Roper Scientific) has a second optical fibre output connected to an illumination device containing galvanometric scanning mirrors (ILAS, Roper Instrument) steered by MetaMorph. It allows precise spatial and temporal control of the focused laser beam at any user-selected region of interest within the sample for targeted photobleaching. Switching between the two fibres for alternating between imaging and bleaching is performed in the millisecond range using a mirror. Oblique illumination acquisition was performed using the 561-nm laser at low power $(300 \mu \mathrm{W}$ at the front of the objective) to precisely image molecules accumulated at the substrate level. After acquiring a 10 -s baseline at $0.5-1 \mathrm{~Hz}$ frame rate, rapid selective photobleaching of two to three synapses was achieved at higher laser power ( $3 \mathrm{~mW}$ at the front of the objective) during $200-300 \mathrm{~ms}$. Fluorescence recovery was then recorded immediately after the bleach sequence for $30 \mathrm{~min}$ at a $0.5-1 \mathrm{~Hz}$ frame rate. Observational photobleaching was kept very low, as assessed by observing control synapses nearby. Data were plotted as normalized fluorescence intensity versus time and fitted by the formula ${ }^{44}: \phi\left[1-\operatorname{erf}\left(1 /\left(2 \sqrt{k_{\text {diff }}}\right)\right]+\right.$ $(1-\phi)\left[1-\exp \left(-k_{\text {reac }} t\right)\right]$, where $\phi$ is the fraction of synaptic Nlg1 or LRRMT2 molecules diffusing freely with coefficient $D, k_{\text {diff }}\left(\right.$ in $\min ^{-1}$ ) is a characteristic diffusive rate equal to $D / r^{2}$ (where $r$ is the radius of the bleached area), $(1-\phi)$ the fraction of molecules trapped in adhesive interactions with presynaptic $\operatorname{Nrx} 1 \beta$ and $k_{\text {reac }}\left(\right.$ in $\min ^{-1}$ ) the turnover rate of bound molecules. In this model, the synaptic enrichment of Nlg1 or LRRTM2 at synapses is equal to the total number of molecules (bound + free) versus free molecules, that is, the ratio $1 / \phi$. In control experiments, FRAP was performed on DIV 15 neurons electroporated with Nlg1GFP using a 488-nm laser power of either 0.4 or $4 \mathrm{~mW}$ at the front of the objective.

Confocal microscopy. Single electroporated neurons from organotypic slice culture co-expressing GFP, BirA ${ }^{\mathrm{ER}}$ and AP-Nlg1 were surface-labelled with mSA-Atto $647 \mathrm{~N}$, biotin antibody-Atto594 or streptavidin-Atto $647 \mathrm{~N}$ in artificial cerebrospinal fluid (ACSF) (in mM, $125 \mathrm{NaCl}, 2.5 \mathrm{KCl}, 2 \mathrm{CaCl}_{2}, 1 \mathrm{MgCl}_{2}, 25 \mathrm{NaHCO}_{3}, 1.25$ $\mathrm{NaH}_{2} \mathrm{PO}_{4}$ and 25 glucose, $\mathrm{pH} 7.4$ ) for $10 \mathrm{~min}$ or $1 \mathrm{~h}$, rinsed and fixed with $4 \% \mathrm{PFA}-$ $0.2 \%$ glutaraldehyde in PBS-BSA $1 \%$ for $2 \mathrm{~h}$ at $4{ }^{\circ} \mathrm{C}$. Images were acquired on a commercial Leica DMI6000 TCS SP5 microscope using a $\times 63,1.4$ NA oil objective and a pinhole opened to one time the Airy disk. Images of $512 \times 512$ pixels were acquired at a scanning frequency of $400 \mathrm{~Hz}$.

Statistics. Statistical values are given as mean \pm s.e.m., unless otherwise stated. Statistical significance was calculated using GraphPad Prism. All data sets comparing two conditions were tested by the non-parametric Mann-Whitney test. Data sets containing more than two conditions were compared by one-way analysis of variance test, followed by a post hoc Dunn's test. Sample size was based on two to three different cultures per condition, 2-10 cells per experiment. Randomization of samples was performed for all experiments. When critical comparison of different labelling conditions was involved, experiments and image analysis were performed blindly.

\section{References}

1. Triller, A. \& Choquet, D. New concepts in synaptic biology derived from single-molecule imaging. Neuron 59, 359-374 (2008).

2. Willig, K. I. et al. Nanoscale resolution in GFP-based microscopy. Nat. Methods 3, 721-723 (2006).

3. Manley, S. et al. High-density mapping of single-molecule trajectories with photoactivated localization microscopy. Nat. Methods 5, 155-157 (2008).

4. Shroff, H., Galbraith, C. G., Galbraith, J. A. \& Betzig, E. Live-cell photoactivated localization microscopy of nanoscale adhesion dynamics. Nat. Methods 5, 417-423 (2008). 
5. Dani, A., Huang, B., Bergan, J., Dulac, C. \& Zhuang, X. Superresolution imaging of chemical synapses in the brain. Neuron 68, 843-856 (2010).

6. Heilemann, M. et al. Subdiffraction-resolution fluorescence imaging with conventional fluorescent probes. Angew. Chem.- Int. Ed. Engl. 47, 6172-6176 (2008).

7. Sharonov, A. \& Hochstrasser, R. M. Wide-field subdiffraction imaging by accumulated binding of diffusing probes. Proc. Natl Acad. Sci. USA 103, 18911-18916 (2006).

8. Giannone, G. et al. Dynamic superresolution imaging of endogenous proteins on living cells at ultra-high density. Biophys. J. 99, 1303-1310 (2010).

9. Huang, B., Babcock, H. \& Zhuang, X. Breaking the diffraction barrier: super-resolution imaging of cells. Cell 143, 1047-1058 (2010).

10. Fernández-Suárez, M. \& Ting, A. Y. Fluorescent probes for super-resolution imaging in living cells. Nat. Rev. Mol. Cell Biol. 9, 929-943 (2008).

11. Deschout, H. et al. Precisely and accurately localizing single emitters in fluorescence microscopy. Nat. Methods 11, 253-266 (2014).

12. Lau, L., Lee, Y. L., Sahl, S. J., Stearns, T. \& Moerner, W. E. STED microscopy with optimized labeling density reveals 9 -fold arrangement of a centriole protein. Biophys. J. 102, 2926-2935 (2012).

13. Ries, J., Kaplan, C., Platonova, E., Eghlidi, H. \& Ewers, H. A simple, versatile method for GFP-based super-resolution microscopy via nanobodies. Nat. Methods 9, 582-584 (2012).

14. Rothbauer, U. et al. Targeting and tracing antigens in live cells with fluorescent nanobodies. Nat. Methods 3, 887-889 (2006).

15. Van Bockstaele, F., Holz, J.-B. \& Revets, H. The development of nanobodies for therapeutic applications. Curr. Opin. Investig. Drugs 10, 1212-1224 (2009).

16. Rossier, O. et al. Integrins $\beta(1)$ and $\beta(3)$ exhibit distinct dynamic nanoscale organizations inside focal adhesions. Nat. Cell Biol. 14, 1057-1067 (2012).

17. Liu, D. S., Phipps, W. S., Loh, K. H., Howarth, M. \& Ting, A. Y. Quantum dot targeting with lipoic acid ligase and HaloTag for single-molecule imaging on living cells. ACS Nano 6, 11080-11087 (2012).

18. Xue, L., Karpenko, I. A., Hiblot, J. \& Johnsson, K. Imaging and manipulating proteins in live cells through covalent labeling. Nat. Chem. Biol. 11, 917-923 (2015).

19. Sekine-Aizawa, Y. \& Huganir, R. L. Imaging of receptor trafficking by using alpha-bungarotoxin-binding-site-tagged receptors. Proc. Natl Acad. Sci. USA 101, 17114-17119 (2004).

20. Grunwald, C. et al. Quantum-yield-optimized fluorophores for site-specific labeling and super-resolution imaging. J. Am. Chem. Soc. 133, 8090-8093 (2011).

21. Liu, D. S., Loh, K. H., Lam, S. S., White, K. a \& Ting, A. Y. Imaging trans-cellular neurexin-neuroligin interactions by enzymatic probe ligation. PLoS ONE 8, e52823 (2013).

22. Stadler, C. et al. Immunofluorescence and fluorescent-protein tagging show high correlation for protein localization in mammalian cells. Nat. Methods 10, 315-323 (2013)

23. Howarth, M., Takao, K., Hayashi, Y. \& Ting, A. Y. Targeting quantum dots to surface proteins in living cells with biotin ligase. Proc. Natl Acad. Sci. USA 102, 7583-7588 (2005).

24. Howarth, M. et al. A monovalent streptavidin with a single femtomolar biotin binding site. Nat. Methods 3, 267-273 (2006).

25. Lim, K. H., Huang, H., Pralle, A. \& Park, S. Engineered streptavidin monomer and dimer with improved stability and function. Biochemistry 50, 8682-8691 (2011).

26. Demonte, D., Drake, E. J., Lim, K. H., Gulick, A. M. \& Park, S. Structure-based engineering of streptavidin monomer with a reduced biotin dissociation rate. Proteins 81, 1621-1633 (2013).

27. Strassberger, V., Trüssel, S., Fugmann, T., Neri, D. \& Roesli, C. A novel reactive ester derivative of biotin with reduced membrane permeability for in vivo biotinylation experiments. Proteomics 10, 3544-3548 (2010).

28. Fairhead, M., Krndija, D., Lowe, E. D. \& Howarth, M. Plug-and-play pairing via defined divalent streptavidins. J. Mol. Biol. 426, 199-214 (2014).

29. Jones, S. a, Shim, S.-H., He, J. \& Zhuang, X. Fast, three-dimensional super-resolution imaging of live cells. Nat. Methods 8, 499-508 (2011).

30. Klein, T. et al. Live-cell dSTORM with SNAP-tag fusion proteins. Nat. Methods 8, 7-9 (2011).

31. Mondin, M. et al. Neurexin-neuroligin adhesions capture surface-diffusing AMPA receptors through PSD-95 scaffolds. J. Neurosci. 31, 13500-13515 (2011).

32. Giannone, G. et al. Neurexin-1 $\beta$ binding to neuroligin-1 triggers the preferential recruitment of PSD-95 versus gephyrin through tyrosine phosphorylation of neuroligin-1. Cell Rep. 3, 1996-2007 (2013).

33. Czöndör, K. et al. Unified quantitative model of AMPA receptor trafficking at synapses. Proc. Natl Acad. Sci. USA 109, 3522-3527 (2012).

34. Fu, Y. \& Huang, Z. J. Differential dynamics and activity-dependent regulation of alpha- and beta-neurexins at developing GABAergic synapses. Proc. Natl Acad. Sci. USA 107, 22699-22704 (2010).
35. Neupert, C. et al. Regulated dynamic trafficking of neurexins inside and outside of synaptic terminals. J. Neurosci. 35, 13629-13647 (2015).

36. Tomita, S., Fukata, M., Nicoll, R. a \& Bredt, D. S. Dynamic interaction of stargazin-like TARPs with cycling AMPA receptors at synapses. Science 303, 1508-1511 (2004).

37. Opazo, P. et al. CaMKII triggers the diffusional trapping of surface AMPARs through phosphorylation of stargazin. Neuron 67, 239-252 (2010).

38. Tsetsenis, T., Boucard, a. a., Arac, D., Brunger, a. T. \& Sudhof, T. C. Direct visualization of trans-synaptic neurexin-neuroligin interactions during synapse formation. J. Neurosci. 34, 15083-15096 (2014).

39. Saint-Michel, E., Giannone, G., Choquet, D. \& Thoumine, O. Neurexin/ neuroligin interaction kinetics characterized by counting single cell-surface attached quantum dots. Biophys. J. 97, 480-489 (2009).

40. Beattie, E. C. et al. Regulation of AMPA receptor endocytosis by a signaling mechanism shared with LTD. Nat. Neurosci. 3, 1291-1300 (2000).

41. Ko, J., Fuccillo, M. V, Malenka, R. C. \& Südhof, T. C. LRRTM2 functions as a neurexin ligand in promoting excitatory synapse formation. Neuron $\mathbf{6 4}$, 791-798 (2009).

42. Siddiqui, T. J., Pancaroglu, R., Kang, Y., Rooyakkers, A. \& Craig, A. M. LRRTMs and neuroligins bind neurexins with a differential code to cooperate in glutamate synapse development. J. Neurosci. 30, 7495-7506 (2010)

43. de Wit, J. et al. LRRTM2 interacts with Neurexin 1 and regulates excitatory synapse formation. Neuron 64, 799-806 (2009).

44. Thoumine, O., Lambert, M., Mège, R.-M. \& Choquet, D. Regulation of $\mathrm{N}$-cadherin dynamics at neuronal contacts by ligand binding and cytoskeletal coupling. Mol. Biol. Cell 17, 862-875 (2006).

45. Hughes, L. D., Rawle, R. J. \& Boxer, S. G. Choose your label wisely: water-soluble fluorophores often interact with lipid bilayers. PLOS ONE 9 , e87649 (2014).

46. de Boer, E. et al. Efficient biotinylation and single-step purification of tagged transcription factors in mammalian cells and transgenic mice. Proc. Natl Acad. Sci. USA 100, 7480-7485 (2003).

47. Biermann, B. et al. Imaging of molecular surface dynamics in brain slices using single-particle tracking. Nat. Commun. 5, 3024 (2014).

48. Mahler, B. et al. Towards non-blinking colloidal quantum dots. Nat. Mater. 7, 659-664 (2008).

49. Wu, S. et al. Non-blinking and photostable upconverted luminescence from single lanthanide-doped nanocrystals. Proc. Natl Acad. Sci. USA 106, 10917-10921 (2009)

50. Gokce, O. \& Sudhof, T. C. Membrane-tethered monomeric neurexin LNS-domain triggers synapse formation. J. Neurosci. 33, 14617-14628 (2013).

51. Budreck, E. C. et al. Neuroligin-1 controls synaptic abundance of NMDA-type glutamate receptors through extracellular coupling. Proc. Natl Acad. Sci. USA 110, 725-730 (2013).

52. Soler-Llavina, G. J., Fuccillo, M. V, Ko, J., Sudhof, T. C. \& Malenka, R. C. The neurexin ligands, neuroligins and leucine-rich repeat transmembrane proteins, perform convergent and divergent synaptic functions in vivo. Proc. Natl Acad. Sci. USA 108, 16502-16509 (2011).

53. Chubykin, A. a et al. Activity-dependent validation of excitatory versus inhibitory synapses by neuroligin-1 versus neuroligin-2. Neuron 54, 919-931 (2007).

54. Ko, J., Soler-Llavina, G. J., Fuccillo, M. V, Malenka, R. C. \& Südhof, T. C. Neuroligins/LRRTMs prevent activity- and Ca2 + /calmodulin-dependent synapse elimination in cultured neurons. J. Cell Biol. 194, 323-334 (2011).

55. Peixoto, R. T. et al. Transsynaptic signaling by activity-dependent cleavage of neuroligin-1. Neuron 76, 396-409 (2012).

56. Bemben, M. a et al. CaMKII phosphorylation of neuroligin-1 regulates excitatory synapses. Nat. Neurosci. 17, 56-64 (2014).

57. Tracy, T. E., Yan, J. J. \& Chen, L. Acute knockdown of AMPA receptors reveals a trans-synaptic signal for presynaptic maturation. EMBO J. 30, 1577-1592 (2011).

58. Aoto, J., Martinelli, D. C., Malenka, R. C., Tabuchi, K. \& Südhof, T. C. Presynaptic neurexin-3 alternative splicing trans-synaptically controls postsynaptic AMPA receptor trafficking. Cell 154, 75-88 (2013).

59. Nair, D. et al. Super-resolution imaging reveals that AMPA receptors inside synapses are dynamically organized in nanodomains regulated by PSD95. J. Neurosci. 33, 13204-13224 (2013).

60. Desbois, M., Cook, S. J., Emmons, S. W. \& Bülow, H. E. Directional transsynaptic labeling of specific neuronal connections in live animals. Genetics 200 697-705 (2015).

61. Galland, R. et al. 3D high- and super-resolution imaging using single-objective SPIM. Nat. Methods 12, 641-644 (2015).

62. Dresbach, T., Neeb, A., Meyer, G., Gundelfinger, E. D. \& Brose, N. Synaptic targeting of neuroligin is independent of neurexin and SAP90/PSD95 binding. Mol. Cell Neurosci. 27, 227-235 (2004).

63. Studier, F. W. Protein production by auto-induction in high density shaking cultures. Protein Expr. Purif. 41, 207-234 (2005) 
64. Kaech, S. \& Banker, G. Culturing hippocampal neurons. Nat. Protoc. 1, 2406-2415 (2006).

65. Dean, C. et al. Neurexin mediates the assembly of presynaptic terminals. Nat. Neurosci. 6, 708-716 (2003).

66. Stoppini, L., Buchs, P. A. \& Muller, D. A simple method for organotypic cultures of nervous tissue. J. Neurosci. Methods 37, 173-182 (1991).

67. Izeddin, I. et al. Wavelet analysis for single molecule localization microscopy. Opt. Express 20, 2081-2095 (2012).

68. Kechkar, A., Nair, D., Heilemann, M., Choquet, D. \& Sibarita, J. B. Real-time analysis and visualization for single-molecule based super-resolution microscopy. PLoS ONE 8, e62918 (2013).

69. Hajj, B. et al. Whole-cell, multicolor superresolution imaging using volumetric multifocus microscopy. Proc. Natl Acad. Sci. USA 111, 17480-17485 (2014).

\section{Acknowledgements}

We thank D. Perrais, M. Missler, S. Okabe, P. Scheiffele, T. Dresbach and A. Ting for the generous gift of DNA plasmids; F. Neca and Z. Karatas for molecular biology; the Cell culture facility of the Institute (especially E. Verdier and P. Durand); the animal facility of the University of Bordeaux (in particular A. Lacquemant and A. Gautier); the biochemistry facility of Bordeaux Neurocampus; J. Gilardin for the technical support; G. Giannone and O. Rossier for helpful advice and preliminary experiments; the Bordeaux Imaging Center, in particular P. Legros for STED microscopy; and C. O'Hare for critical reading of the initial manuscript. This work received funding from the Centre National de la Recherche Scientifique, Agence Nationale pour la Recherche (grants Synapse-2Dt, Nanodom and SynAdh), Conseil Régional Aquitaine, Fondation pour la Recherche Médicale and the national infrastructure France BioImaging (grant ANR-10INBS-04-01)

\section{Author contributions}

I.C., M.S. and O.T. designed research and wrote the article. O.T. did initial proof-ofconcept experiments and I.C. performed all subsequent imaging experiments. M.L. provided electroporated organotypic brain slices. C.B. and I.C. performed dSTORM experiments and analysis. B.T. and I.G. generated DNA constructs. B.T. performed biochemistry experiments. K.H.L. and S.P. provided the initial constructs of mSA. I.G. I.C. and M.S. produced and labelled protein probes. J.-B.S. provided the STORM set-up and software for single-molecule analysis. M.S. and O.T. coordinated and oversaw the research project. All authors discussed the results and commented on the manuscript.

\section{Additional information}

Supplementary Information accompanies this paper at http://www.nature.com/ naturecommunications

Competing financial interests: The authors declare no competing financial interests.

Reprints and permission information is available online at http://npg.nature.com/ reprintsandpermissions/

How to cite this article: Chamma, I. et al. Mapping the dynamics and nanoscale organization of synaptic adhesion proteins using monomeric streptavidin. Nat. Commun. 7:10773 doi: 10.1038/ncomms10773 (2016).

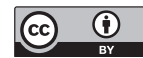

This work is licensed under a Creative Commons Attribution 4.0 International License. The images or other third party material in this article are included in the article's Creative Commons license, unless indicated otherwise in the credit line; if the material is not included under the Creative Commons license, users will need to obtain permission from the license holder to reproduce the material. To view a copy of this license, visit http://creativecommons.org/licenses/by/4.0/ 